Received 00th January 20xx, Accepted 00th January 20xx DOI: $10.1039 / x 0 x \times 00000 x$

\section{Chirality in porous self-assembled monolayer networks at liquid/solid interfaces: induction, reversion, recognition and transfer}

\author{
Yoshito Tobe, ${ }^{* a, b, c}$ Kazukuni Tahara, ${ }^{\text {a,d }}$ Steven De Feyter
}

\begin{abstract}
Chirality in two-dimensions (2D) has attracted increasing attention with regard to interesting fundamental aspects as well as potential applications. This article reports several aspects of supramolecular chirality control as exemplified by selfassembled monolayer networks (SAMNs) formed by a class of chiral building blocks consisting of triangular conjugated core and alkoxy chains on its periphery. It highlights 2D chirality induction phenomena through a classic "sergeants-and-soldiers" mechanism, in which the inducer is incorporated into a network component, as well as through a "supramolecular hostguest" mechanism, in which the inducer is entrapped in the porous space, leading to counterintuitive chirality reversal. Stereochemical control can be extended to three dimensions too, based on interlayer hydrogen bonding of the same class of building block bearing hydroxy groups, exhibiting diastereospecific bilayer formation in both single molecular level and supramolecular orientation between the top and bottom layers. Finally, we showcase that homochiral SAMNs can also be used as templates for grafting of in-situ generated aryl radicals, by covalent bond formation to the basal graphitic surface, thereby yielding topologically chiral functionalized graphite, and thus extending the potential of chiral SAMNs.
\end{abstract}

\section{Introduction}

Chirality is not only an important subject of stereochemistry but also it is relevant to many phenomena in physics, chemistry and biology. However, most of chemical studies focus on three-dimensional systems, namely liquid solutions and solid crystals. Recently surfaces which express chirality in two-dimensions (2D) have attracted increasing attention not only thanks to the plethora of interesting fundamental aspects that are (to be) discovered, ${ }^{1-6}$ but also because of applications in chiral separation, ${ }^{6-8}$ enantioselective heterogeneous catalysis ${ }^{5,9-11}$ and chiroptical materials. ${ }^{12-14}$ Chirality can be bestowed to solid surfaces by several different approaches. ${ }^{5,6}$ Intrinsically chiral surfaces can be found naturally in bulk materials such as quartz and calcite or in single crystal surfaces of achiral materials created by cutting along low symmetry directions. ${ }^{6}$ Another approach is to decorate or modify achiral surfaces by the irreversible adsorption (chemisorption) of chiral molecules, resulting in processes called chiral templating or chiral imprinting, respectively. ${ }^{5,6}$ In the chiral templating process, adsorption of chiral molecules simply renders an achiral surface chiral, ${ }^{1-3}$ whereas in

\footnotetext{
a. Division of Frontier Materials Science, Graduate School of Engineering Science,

Osaka University, Toyonaka, Osaka 560-8531, Japan

b. The Institute of Scientific and Industrial Research, Osaka University, Ibaraki, Osaka 567-0047, Japan

Department of Applied Chemistry, National Chiao Tung University, Hsinchu 30010, Taiwan

d. Department of Applied Chemistry, School of Science and Technology, Meiji University, Kawasaki, Kanagawa 214-8571, Japan

e. Division of Molecular Imaging and Photonics, Department of Chemistry, KU Leuven, 3001 Leuven, Belgium
}

chiral imprinting, reconstruction of an achiral surface induced by chiral adsorbates takes place rendering the surface intrinsically chiral. ${ }^{5}$ Alternatively, achiral surfaces can be modified by reversible adsorption (physisorption) of molecules. ${ }^{1-4}$

Because of a number of advantages such as the versatility and efficiency in creating supramolecular assembled monolayer networks (SAMNs) on surfaces, the latter process has attracted significant interest during the last two decades. ${ }^{15}$ In this case, symmetry elements present in molecules such as inversion centres and mirror planes parallel to the surface are lost due to the presence of the surface, at one side. As a result, not only chiral but also prochiral molecules can become chiral once adsorbed on surfaces. This symmetry breaking takes place both at the single molecular level (i.e., point chirality, called footedness in contrast with handedness in chiral molecules $\left.{ }^{16,17}\right)$ and at supramolecular levels arising from chiral arrangements of prochiral molecules (i.e., organizational chirality). Therefore, even highly symmetric molecules which preserve rotational symmetry on surfaces can create chiral self-assemblies upon adsorption on surfaces. Nevertheless, monolayers formed by prochiral molecules always present both mirror image patterns of opposite handedness in equal abundances rendering the surface globally achiral. On the other hand, by using enantiomerically pure molecules as building blocks, only single-handed patterns will be formed rendering the surfaces globally chiral, if chiral elements of the molecules affect the adsorption and self-assembly processes efficiently.

Achiral building blocks can also be used to form globally chiral surfaces, if a chiral dopant is added to make the self-assembled 
monolayers homochiral. ${ }^{2-4,18}$ In most cases, the chiral dopant is incorporated in the monolayer networks of the achiral building blocks to transfer its chirality rendering the surfaces chiral. Similarly, by adding a small excess of one enantiomer to racemates the major enantiomer may suppress the formation of its mirror image pattern through a process governed by the so-called "majority rules". 2,3,19

Chirality information of the surface structure and monolayers formed on can be obtained by using low-energy electron diffraction (LEED) and scanning tunnelling microscopy (STM), ${ }^{1-6}$ the latter being frequently used for SAMNs formed at the liquid-solid interfaces, given the solid is conductive. . $^{15,20,21}$

In this Feature article, we showcase several interesting aspects related to $2 \mathrm{D}$ chirality of porous self-assembled monolayer networks formed at liquid/solid interfaces by triangular building blocks, i.e. dehydro[12]annulenes (DBAs) substituted with six alkoxy chains on the periphery (Chart 1 ), the result of a long-standing collaborative effort by the research groups in Japan and Belgium. ${ }^{22-25}$ Following a brief summary of the key features of SAMNs formed by DBAs, this Feature article first describes the induction and reversion of chirality in the porous 2D networks, followed by a disussion of the stereospecific epitaxial growth of chiral monolayers forming porous bilayers. It concludes by demonstrating the successful transfer of supramolecular 2D network chirality, using the network as template, for the covalent functionalization of the substrate. We present not only how a small difference in local intermolecular interactions may get amplified to define the global chirality of SAMNs, sometimes in a surprising manner, but also demonstrate the potential of chiral SAMNs in applications.

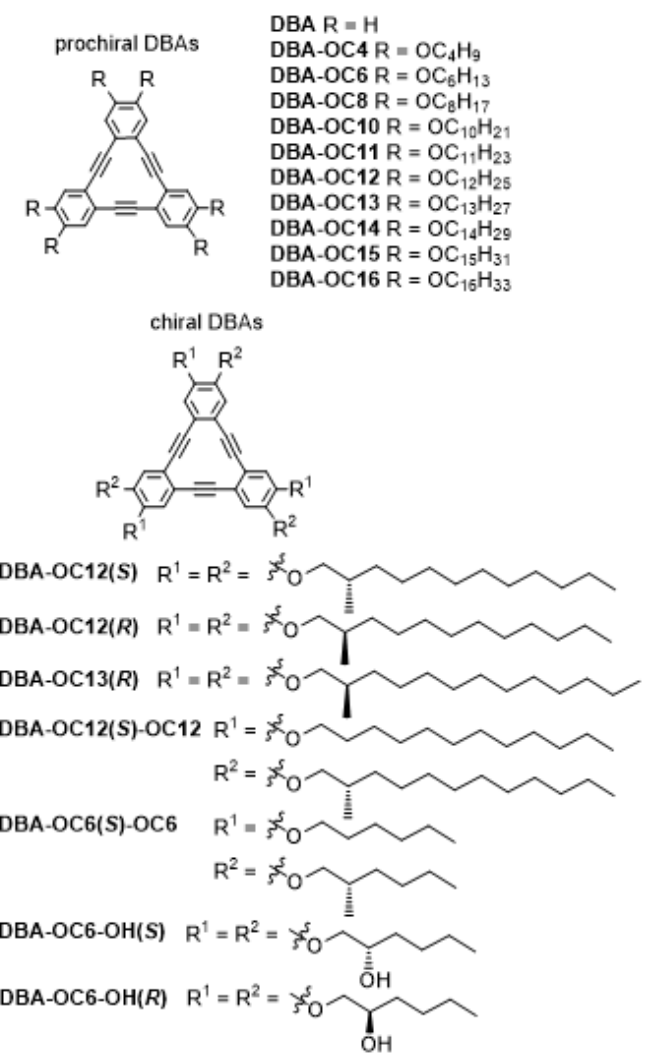

Chart 1 Chemical structures of prochiral DBA and chiral DBA derivatives.

\section{Chiral porous self-assembled networks formed at liquid-solid interfaces}

First, we summarise some key features regarding chirality of SAMNs formed by DBA derivatives.

\subsection{Prochiral DBA derivatives}

Unsubstituted DBA adsorbs on coinage metal surfaces under ultrahigh vacuum (UHV) condition in an epitaxial manner, at the single molecule level, due to van der Waals type molecule-surface interactions. ${ }^{26-28} \mathrm{On} \mathrm{Au}(111)$ and $\mathrm{Ag}(111)$ surfaces two molecular orientations, clockwise (CW) and counter clockwise (CCW), with respect to the (122) axes break the mirror-symmetry planes of the surface, leading to point chirality at the single molecule level. However, because of the existence of two types of hollow sites (fcc and hcp) on the $\mathrm{Ag}(111)$ surface, two pseudo-enantiomeric pairs of DBA appear with respect to the (110) axes, leading to pseudodiastereomeric pairs consisting of the four pseudo-enantiomers (Fig. 1). ${ }^{27}$ 
(a)
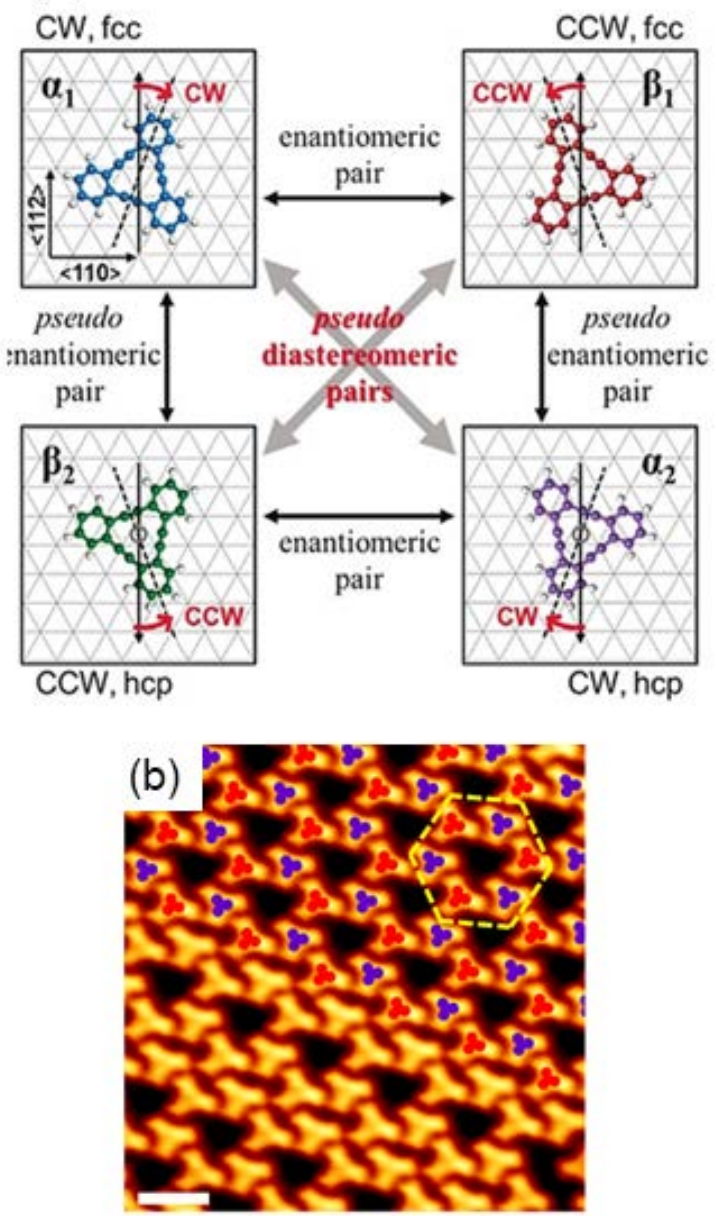

Fig. 1 (a) 2D isomeric relationship of DBA on $\mathrm{Ag}(111)$ surface with respect to (112) and (110) axes leading to pseudo-enantiomeric and pseudo-diastereomeric relations. (b) STM image of a porous SAMN of DBA on $\mathrm{Ag}(111)$ at coverage of $\sim 0.7 \mathrm{ML}$ exhibiting domain-specific organizational chirality composed of only two stereoisomers (colorcoded) of DBA in an alternating manner. Reproduced from Ref 27 with permission from John Wiley and Sons.

While the DBA core itself shows point chirality upon adsorption on some metallic surfaces, and also domain-specific chirality, the main players in this article are DBAs substituted with alkoxy groups: $D_{3 h}$-symmetric DBA-OCn with six identical alkoxy chains, and $C_{3 h^{-}}$ symmetric DBA-OCn-OCm with two different alkoxy chains in an alternating manner, where $\mathrm{n}$ and $\mathrm{m}$ denote the number of carbons in the alkyl groups (Chart 1). Where appropriate, methyl or hydroxy groups are introduced to create stereogenic centres, adjacent to the $\mathrm{OCH}_{2}$ groups of the alkoxy chains to make the building block chiral, as a clue to control the supramolecular chirality of the SAMNs. Though self-assembly of a few DBA-OCns has been studied on metal surfaces either at liquid-solid interfaces ${ }^{29}$ or in UHV, ${ }^{26,30}$ this article focuses on the SAMNs formed at the interface of graphite and organic solvents, typically 1-phenyloctane (PO) and 1,2,4trichlorobenzene (TCB), and occasionally 1-octanoic acid (OA), formed through as driving forces van der Waals interactions between graphite and the alkoxy chains, and between the alkoxy chains of neighbouring molecules, which align parallel by interdigitation to each other. Typically, experiments were done by dropping a dilute $\left(10^{-5}-10^{-7} \mathrm{M}\right)$ solution of the DBA derivatives in one of the above solvents on graphite and STM images at the liquid/solid interface were acquired by immersing a tip into the solution.

Initially, we found that DBA-OC10 through DBA-OC18 with even number alkoxy chains formed two different kinds of SAMNs at the TCB/graphite interface (Fig. 2). ${ }^{31,32}$ One is the hexagonal porous type, in which all six alkoxy chains are adsorbed on the surface forming intermolecular linkages with three neighbouring DBA-OCn molecules (Fig. 2b,d). The vacant areas are filled with co-adsorbed solvent molecules thus stabilizing the system. The other is a close-packed pattern, so-called linear morphology, in which one or two of the alkoxy chains is (are) not adsorbed on the surface and dissolved in the supernatant solution phase (Fig. 2a,c). The DBA-OCn molecules are linked by the interdigitated alkyl chains with the two neighbouring molecules, forming a one-dimensional zig-zag chain. DBAs with longer alkyl chains tend to assemble in the linear form. However, we subsequently revealed that the distribution of the two polymorphs was critically dependent on the type of the solvent, solute concentration ${ }^{33}$ and temperature. ${ }^{34}$ Several different polymorphs in addition to the two above-mentioned structures were observed in OA depending on the length of alkoxy chains and their parity, indicating the delicate balance between the stability of network pattern and solution activity. ${ }^{35}$ However, by optimizing the conditions, we are able to form the porous phase selectively for DBAOCns ranging from DBA-OC4 to DBA-OC20. This made it possible to modify the size of the porous space to accommodate guest molecules in a size and shape selective manner by co-adsorption on the surface. ${ }^{36,37}$ (a)

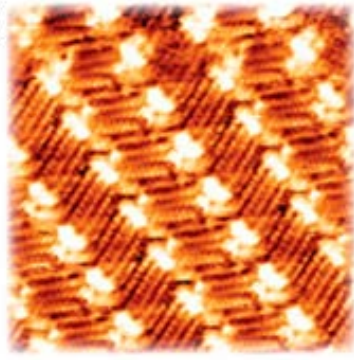

(c)

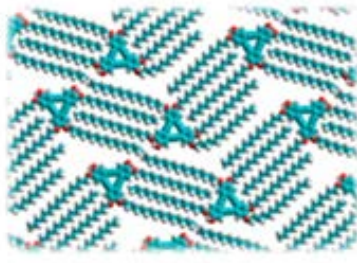

(b)

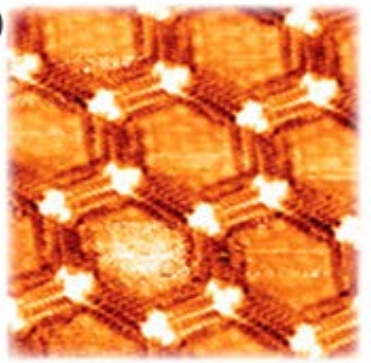

(d)

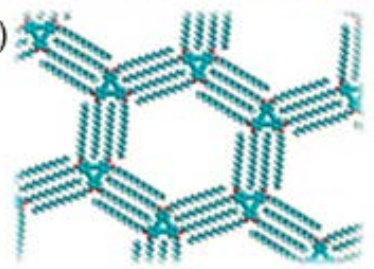

Fig 2 (a) Linear and (b) porous patterns of SAMNs formed by DBAOC16 and (c, d) the corresponding network models. Reproduced 
from Ref 34 with permission. Copyright 2015 American Chemical Society.

A single molecule of a prochiral DBA-OCn put on a flat surface is assumed to remain achiral if it adopts $D_{3 h}$ symmetry with the alkoxy chains orienting straight in a direction perpendicular to the edge of the triangle DBA core as seen in the model in Fig. $2 \mathrm{~d}$. However, when two DBA-OCn molecules are linked by alkyl chain interdigitation to form a dimer, two enantiomeric motifs arise through coupling tentatively called + and - type interdigitation modes as shown in Fig. 3a. If porous networks are formed by repeating either of the enantiomeric linkages in a cyclic manner, the resulting $2 \mathrm{D}$ networks become chiral as well as shown in Fig. 3a. ${ }^{38,39}$ The combination of six identical interdigitation motifs produces a chiral hexagonal unit having inner alkyl chains directing either in CCW or CW direction, which leads to domain specific organizational (supramolecular) chirality as shown in the STM image of a SAMN of DBA-OC12 formed at the $\mathrm{PO} /$ graphite interface (Fig. $3 \mathrm{~b}$ ). The alkyl chains align parallel to the main symmetry axes of graphite, which are defined by the intersections of the three mirror planes of the basal (0001) plane of graphite in the directions that bisect the opposite edges of the hexagonal unit, running parallel with the equivalent $(\overline{1} 2 \overline{1} 0$, crystallographic directions. ${ }^{4}$ The directions of these axes are indicated by double-headed white arrows in Fig. 3b. Needless to say, CCW and CW patterns are formed in equal probability in the absence of chiral biases. It is also possible to form a non-uniform hexagonal unit, for example, by combining DBA-OCn molecules, for example, with two + and four - interdigitation modes as depicted in Fig. 3a bottom. Indeed, there are a total of eight possible hexagonal structures consisting of different interdigitation patterns. ${ }^{38,39}$ Though a variable amount (typically a few percent) of irregular hexagonal patterns are formed by room temperature sample deposition, thermal annealing turns the surface covered solely with the regular hexagonal phase, indicating that the latter is thermodynamically more stable. ${ }^{39}$ Similar to the porous networks, repeating either + or - type interdigitation motif in the linear pattern leads to chiral linear networks. ${ }^{40}$ (a)

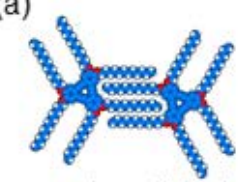

+ type interdigitation
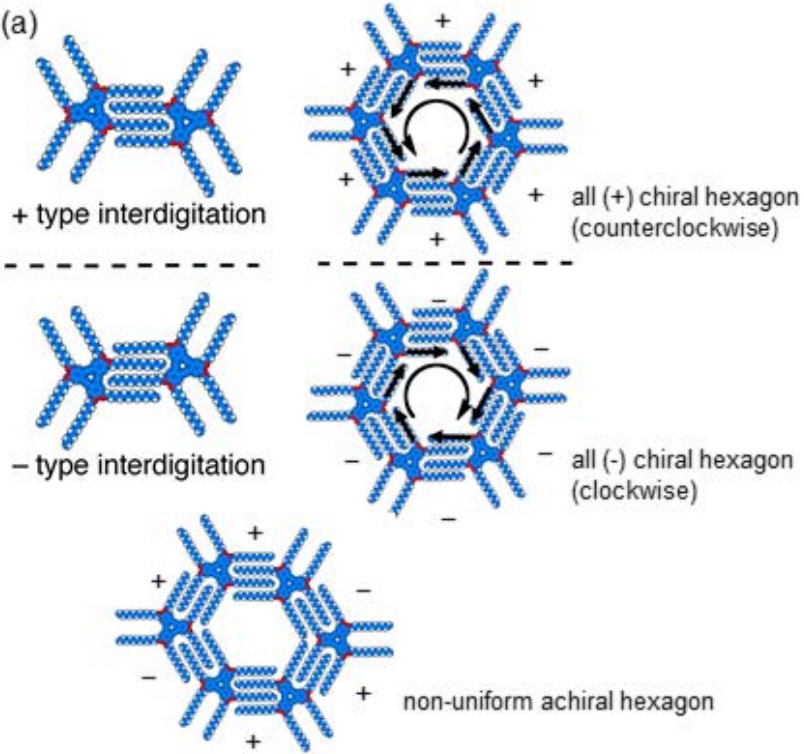

(b)

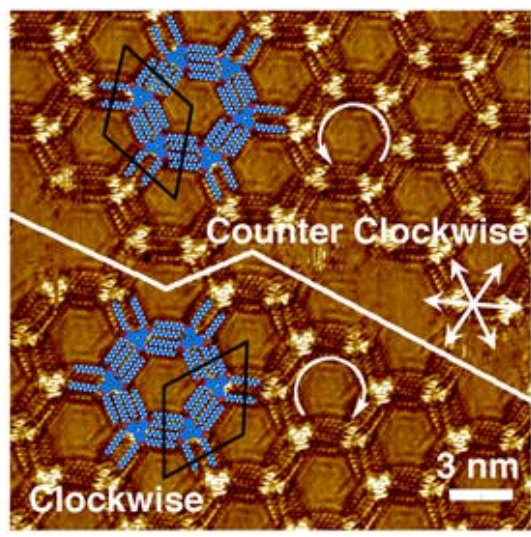

Fig. 3 (a) Molecular models of + and - interdigitation patterns of DBAOC12 and a chiral hexagonal unit formed by all + type interdigitation pattern (top), all - type interdigitation pattern (middle), and a nonuniform hexamer formed by combination of + and - interdigitation motifs (bottom). The black arrows in the chiral nanowell indicate the CCW and CW direction of the inner alkyl chains. (b) STM image of the porous structure of DBA-OC12 at the PO/graphite interface. Molecular models are superimposed on the image. The white line indicates a domain boundary between $\mathrm{CW}$ and $\mathrm{CCW}$ honeycomb structures. The black rhombi and six white arrows indicate the unit cell of the honeycomb structure and the main symmetry axes of graphite, respectively. Reproduced from Ref 24 and 38 with permission from the Chemical Society of Japan and Springer Nature, respectively.

In the models in Fig. 2 and 3, though the alkoxy chains are described to orient perpendicular with respect to the edge of the DBA core for simplicity, it is not fully accurate. Rather they tilt either in a $\mathrm{CW}$ or CCW direction with a tilt angle $\delta$, thereby rendering the DBA-OCn molecule chiral at single molecule level (Fig. 4a). ${ }^{39}$ Because of single molecular and organizational chirality, there are two pairs of diastereomers in the resulting regular hexagonal networks which are $C_{6}$-symmetric. Since the alkoxy chains align parallel with respect 
to the main symmetry axes of graphite to maximize van der Waals interactions, the DBA cores are rotated by specific angles with respect to the graphite lattice similar to the case of DBA on metal surfaces (Fig. 1). As a result, also the unit cell vectors of the SAMNs are tilted with respect to the graphite lattice (expressed as tilt angle $\vartheta$ or $\alpha$ with respect to a main symmetry axis of graphite or a normal of the symmetry axis), which can be determined experimentally from the STM images (Fig. 4b). Interestingly, the tilt angles of DBA-OCns with odd number of alkoxy chains (such as C11, C13 and C15; DBAOCn-odd) are smaller than those with even number alkoxy chains (such as C12, C14 and C14; DBA-OCn-even) as shown in Table 1. The different tilt angles likely originate from a steric mismatch at the end of odd number alkoxy chains confined in the interdigitated alignment due to an unfavourable direction of the terminal C-C bond. In the following discussion, however, the alkoxy chains are assumed to orient to the orthogonal direction, because the tilt angles are small particularly for DBA-OCn-even. As can be seen in Table 1, the tilt angle decreases with decreasing alkoxy chain length for DBA-OCneven, probably because adjusting the mismatch becomes less difficult for shorter alkoxy chains. (a)

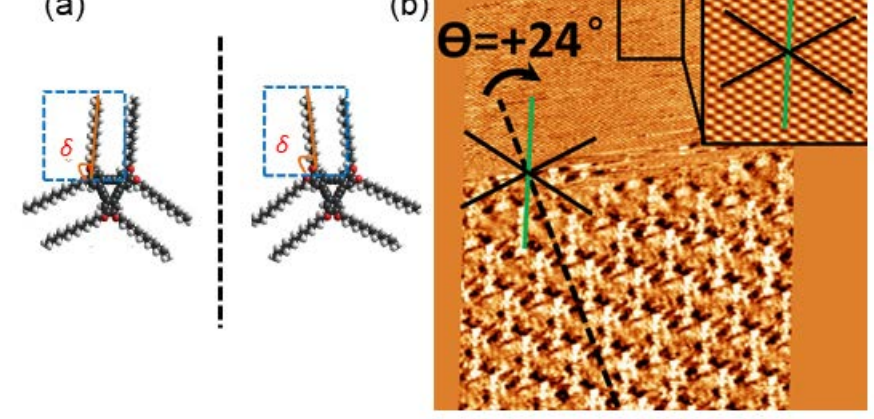

Fig. 4 (a) Molecular models of DBA-OC16 showing the tilt angle $\delta$ between alkoxy chain and the edge of the DBA core. (b) STM image of SAMN of DBA-OC6-OH(R) and underlying graphite indicating a tilt angle $\vartheta$ between a unit cell vector (dotted line) and a symmetry axis of graphite (inset: enlarged image of graphite and the symmetry axes). Reproduced from Ref 39 and 74 with permission. Copyright 2013 and 2020 American Chemical Society.

Table 1. Tilt angles $(\alpha)$ of unit cell vectors of SAMNs of DBA derivatives with respect to a graphite normal.

\begin{tabular}{|l|l|}
\hline DBA derivative & $\alpha$, degree \\
\hline DBA-OC4 & 5.9 \\
\hline DBA-OC6 & 6.3 \\
\hline DBA-OC8 & 8.8 \\
\hline DBA-OC11 & 1.1 \\
\hline DBA-OC12 & 10.3 \\
\hline DBA-OC13 & 1.8 \\
\hline DBA-OC14 & 10.5 \\
\hline DBA-OC15 & 2.8 \\
\hline DBA-OC16 & 10.7 \\
\hline
\end{tabular}

\subsection{Chiral DBA derivatives}

By simply introducing a chiral centre adjacent to the $\mathrm{OCH}_{2}$ unit of the alkoxy chain of DBA-OCn by methyl group, the surface was covered exclusively by SAMNs of either CW or CCW supramolecular chirality. ${ }^{38}$ For example, chiral DBA derivative (CDBA), CDBA-OC12(S), exclusively forms domains of $\mathrm{CW}$ porous structure consisting of type interdigitation (Fig. 5). On the other hand, CDBA-OC13(R) only forms CCW structure with + type interdigitation. The surface chirality was easily assigned by the interdigitation pattern determined from the relative position of two adjacent DBA cores in high resolution STM images (Fig. 3a) or from the orientation of the unit cell vector with respect to a graphite normal (Fig. 4b). Though we recognized fuzzy features in certain images, which were ascribed to cDBA molecules self-occupying a part of the porous space of the network, we were unaware at that time of their amazing role in controlling surface chirality, which is described in section 3 .

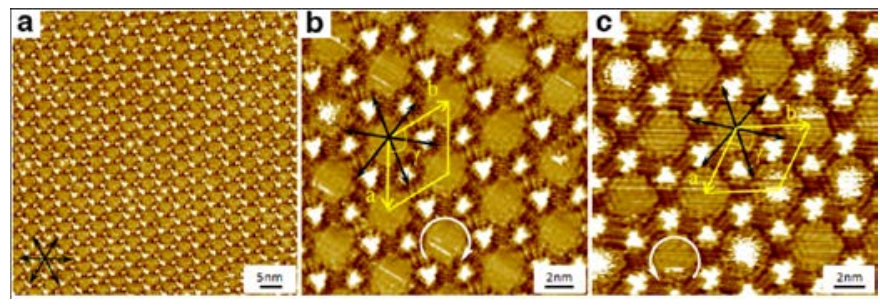

Fig. 5 STM images of SAMNs of CDBAs at the PO/graphite interface. (a) Large scale image and (b) high resolution image of CDBA-OC12(S). (c) High resolution image of CDBA-OC13(R). Unit cells and the main symmetry axes of graphite are indicated by yellow rhombi and black arrows, respectively. Reproduced from Ref 38 with permission from Springer Nature.

The CW handedness of the SAMN formed by CDBA-OC12(S) is explained as follows (Fig. 6). When CDBA-OC12(S) is placed on the surface, three methyl groups will orient away with respect to the surface whereas the other three are forced to orient down (Fig. 6a). ${ }^{41}$ As a result, two different patterns of interdigitation become possible; in the + type interdigitation pattern both methyl groups on the stereogenic centres of the two middle alkyl chains face down to the graphite surface (Fig. 6b), whereas in the - type interdigitation pattern both methyl groups point away from the surface (Fig. 6c). Apparently, it seems more difficult to release steric crowding in the former interdigitation pattern. Indeed, molecular mechanics simulations for the simplified hexamer models indicate that CCW structure consisting of the + type interdigitation is less stable than $\mathrm{CW}$ structure of the - type interdigitation by $9.7 \mathrm{kcal} / \mathrm{mol}$ (Fig. $6 \mathrm{~d}, \mathrm{e}$ ). 
(a)

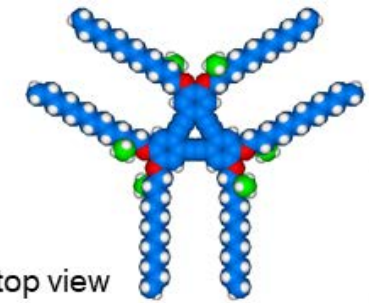

(b)

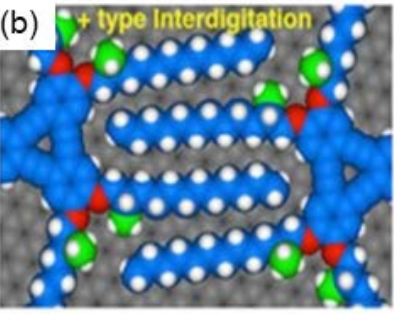

outer Me-up, inner Me-down
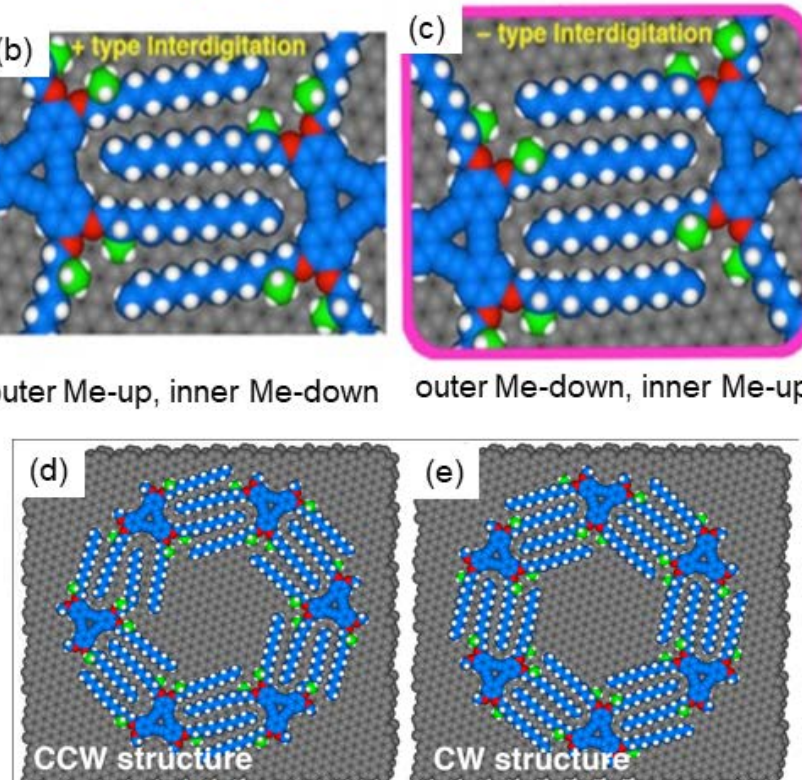

outer Me-down, inner Me-up

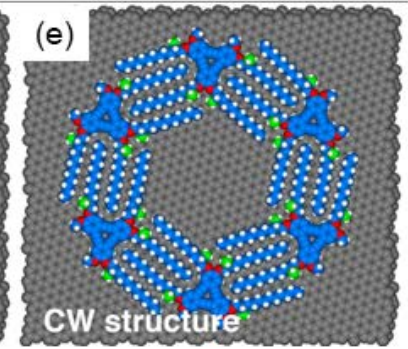

$9.7 \mathrm{kcal} / \mathrm{mol}$ more stable

Fig. 6 (a) Top and side views of a molecular model of cDBA-OC12(S). Methyl carbon atoms are shown in green. (b, c) + and interdigitation modes of alkyl chains, respectively, of a pair of cDBAOC12(S) molecules. (d, e) Model hexamers of CDBA-OC12(S) having CCW and CW structures, respectively, assembled on a bilayered graphene. Reproduced from Ref 24 and 38 with permission from the Chemical Society of Japan and Springer Nature, respectively.

The stereochemical control can be achieved too by only three rather than six stereogenic centres in a molecule, however with a reversed stereochemical outcome. To this end, we investigated SAMN of CDBA-OC12(S)-OC12 with three chiral $C_{12}$ chains each carrying an $S$ stereocentre and three achiral $C_{12}$ chains in an alternating manner at the $\mathrm{PO} /$ graphite interface. ${ }^{42}$ Surprisingly, the surface was covered by CCW pattern of CDBA-OC12(S)-OC12 (94\% after deposition at room temperature and $100 \%$ after annealing), contrary to CDBA-OC12(S) which formed CW structure exclusively. For CDBA-OC12(S)-OC12, since all methyl groups are expected to point away from the graphite surface, the two methyl groups in an interdigitated CDBA dimer will be located at either two inner alkyl chains (by - type interdigitation in CW network) or two outer alkyl chains (by + type interdigitation in CCW network). Molecular mechanics simulations using models similar to those in Fig. $6 \mathrm{~d}$,e indicated the latter geometry is preferred by $6.8 \mathrm{kcal} / \mathrm{mol}$ due to more favourable van der Waals interactions between the alkyl chains, revealing that a subtle stereochemical modification at single molecular level leads a drastic change in the outcome at supramolecular level.

\section{Induction of chirality through sergeants-and- soldiers mechanism}

It has been established by Green and co-workers in their study on macromolecular assemblies in solution that a small number of chiral molecules (sergeants) can bias the handedness of the supramolecular assemblies of achiral building blocks (soldiers). ${ }^{43,44}$ This protocol known as the "sergeants-and-soldiers" principle has been frequently used to control supramolecular chirality of various types of self-assemblies of organic molecules in solution. In many cases, chiral inducers are structurally resembled derivatives of achiral building blocks and incorporated into supramolecular assemblies to define chirality. However, only a few such examples have been reported in 2D assemblies formed on metal surfaces, ${ }^{45-47}$ as well as at liquid/solid interfaces. ${ }^{48,49}$ Excluding the chiral solvents ${ }^{50-52}$ and structurally irrelevant additives, ${ }^{53}$ none was known except for the cDBAs. We demonstrated that this method was effectively used for the porous assembly of DBA-OC12 by using CDBA-OC12(S) or $\operatorname{CDBA-OC12(R)}$ as a chiral inducer at the $\mathrm{PO} /$ graphite interface. ${ }^{38,54}$ Our initial experiments performed at room temperature deposition, however, showed rather inefficient induction ability; 10 mol\% of CDBA-OC12(S) yielded a surface with 91\% CW pattern (the same chirality as that formed by the chiral inducers alone) at $C=5.0 \times 10^{-7} \mathrm{M}$. Fig. 7 shows that cDBA molecules are incorporated in the network as a building block, thereby controlling the handedness of the domain. However, $\sim 30$ mol\% of the inducer was necessary to achieve complete single handedness.

One of the reasons we initially attributed this low efficiency to is the lower affinity of the CDBA molecule compared to achiral DBA toward adsorption on graphite. This was believed due to unfavourable interactions caused by the methyl groups orienting down toward the graphite surface, since the proportion of CDBA molecules observed in the network was much less than that in the solution as shown in Fig. 7. Counting the number of adsorbed cDBA was possible, thanks to a dark rim surrounding the triangle $D B A$ core of CDBAs, thus enabling discrimination of CDBA from achiral DBA adsorbed on graphite. Based on the simulation using density functional theory calculations, the origin of the dark rim was attributed to the lack of local density of states near the stereogenic centre at low sample bias. ${ }^{55}$ The other reason for the inefficient chirality induction was attributed to the small energy difference between the $\mathrm{CW}$ and $\mathrm{CCW}$ networks in the heteromolecular pairs compared to the homomolecular pairs in combination with a kinetic effect, inhibiting the formation of thermodynamically equilibrated phases. Indeed, we found later that by annealing the solution of DBAOC12 containing just $10 \mathrm{~mol} \%$ of CDBA-OC12(S) at $80^{\circ} \mathrm{C}$ a homochiral $\mathrm{CW}$ pattern was formed accompanying coalescence of domains. ${ }^{54}$ Careful inspection showed that under those conditions CDBA molecules were not included in the network after annealing, indicating that the homochiral surface was entirely composed of achiral DBA molecules. This represents an example of chiral memory effect, ${ }^{53,56}$ which arises in this case from the lower adsorption energy of CDBA-OC12(S) compared to DBA-OC12, the former desorbing preferentially during the annealing step. These results indicate that 
only a small number of inducer molecules are necessary to define the chirality of whole domain, indicating a high chirality induction efficiency in this system through the sergeants-and-soldiers mechanism. cDBA-OC12(S)-OC12 with three stereocentres also serves as a chiral inducer for achiral CDBA-OC12 to the antipodal CCW pattern yet with somewhat lower efficiency compared to CDBA$\operatorname{OC12}(S) .42$
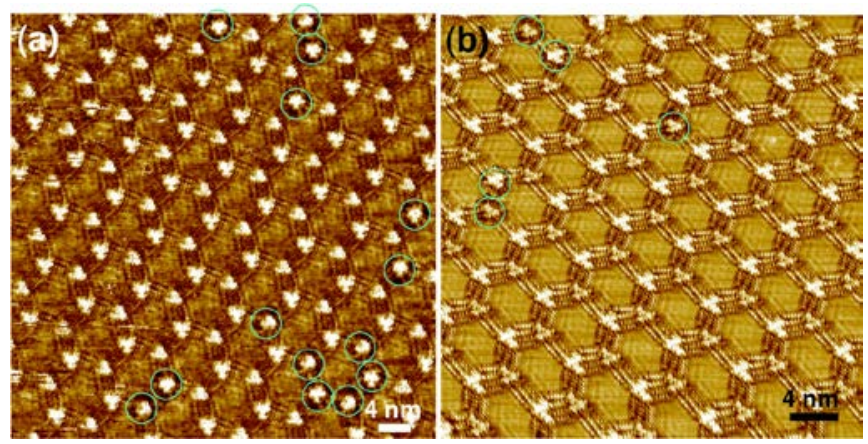

Fig. 7 (a, b) STM images of the CW porous structures formed by a mixture of CDBA-OC12-(S) and DBA-OC12 at 1:1 (a) and 9:1 (b) mixing ratio. The DBAs with dark rims highlighted by green circles are CDBAOC12-(S) molecules. The population of the CDBA molecule on the surface is much less than that of solution composition, for example $4 \%$ in the case of (b). Reproduced from Ref 38 with permission from Springer Nature.

Chiral inducers are not limited to CDBA-OC12(S) which resembles the achiral supramolecular building blocks. In line with results obtained for other systems, ${ }^{50-52}$ chiral solvents also serve as inducer of chirality for DBA networks at the PO/graphite interface. ${ }^{57,58}$ For this purpose, achiral DBA-OCn-evens of different alkoxy chain length $n=6-16$ were examined. As chiral solvents, a series of aliphatic alcohols with different chain lengths bearing an $\mathrm{OH}$ group at the stereogenic 2-position were used. When DBA-OC10 was used as a building block, among the chiral solvents with carbon number ranging from 6 to 12 except for 2-octanol and 2-decanol exhibited the highest induction of chirality (> ca. $90 \%$ of homochiral surface). Conversely, when 2-octanol was used as a solvent, nearly perfect chirality induction was observed for DBA-OC6 and DBA-OC8, whereas the efficiency decreases monotonically with increasing alkoxy chain length of DBA-OCn-evens as shown in Fig. 8. These results clearly indicate that matching the alkyl chain lengths of the building block and solvent is an important aspect for chirality induction. Based on the experimental results coupled with molecular dynamics simulations, several mechanisms for chirality induction were proposed. Among them two, which are consistent with the key role played by solvent-DBA interactions, are thought to be compelling although not conclusive. These are (1) the formation of a DBA-solvent precursor complex that biases a specific handedness of the porous network and (2) the formation a solvent layer on the surface prior to DBA adsorption from which solvent molecules are replaced by DBA molecules.

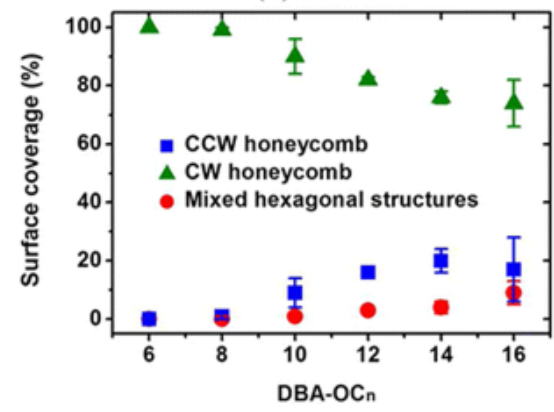

Fig. 8 Alkoxy chain length dependence for 2D chirality induction of OBA-OCn-evens by $(R)$-2-octanol showing $\mathrm{CW}$ (green triangle) and CCW (blue square) patterns along with mixed distorted hexagonal structures. Reproduced with permission from Ref. 57. Copyright 2012 American Chemical Society.

\section{Reversal of chirality via supramolecular host- guest mechanism}

Before describing the supramolecular chirality reversal, we must mention about a curious phenomenon, which we refer to as autoenantioselective adsorption (herein called pseudo-autoadsorption). ${ }^{59}$ By room temperature deposition, a solution of 30 mol\% cDBA-OC12(S) and 70 mol\% DBA-OC12 formed at the $\mathrm{PO} /$ graphite interface mainly the $\mathrm{CW}$ pattern (95\%). Note that the total concentration $\left(2.5 \times 10^{-6} \mathrm{M}\right)$ was slightly higher than the experiment discussed above $\left(5 \times 10^{-7} \mathrm{M}\right)$. As shown in Fig. 9a (bottom), in the CW network CDBA-OC12(S) molecules, which are identified by the dark rim around the triangular DBA core, serve as chirality inducers through the sergeants-and-soldiers mechanism as described above. Whereas in the $\mathrm{CW}$ domain most of the network pores are empty, we realized that in the CCW domain (top left) most of the pores are filled by fuzzy features, which we attribute to cDBAOC12(S) molecules folded in windmill-like conformation (Fig. 9b). Statistical analysis indicates that only $34 \%$ of the CCW pores are empty in contrast to $85 \%$ of the pores in the $\mathrm{CW}$ domains. The windmill-like conformation of the guest CDBA-OC12(S) is chiral, because its alkyl chains are folded in a CW direction to make the three methyl groups orient up with respect to the graphite surface to avoid unfavourable interactions (Fig. 9c). The different probability of occupation of the guest in the $\mathrm{CW}$ and $\mathrm{CCW}$ domains must be due to the different stability between these host-guest systems. Indeed, the MM simulations using hexamer models indicates that CDBA-OC12(S) in the CCW pattern is more favourable than in the CW form by 6 $\mathrm{kcal} / \mathrm{mol}$ in agreement with the experimental results. The difference arises from the better fit of the chiral windmill-shape of the guest in the $\mathrm{CCW}$ porous space rather than the $\mathrm{CW}$ one. This observation also suggests that CDBA-OC12(S) preferentially occupies the porous space of the network over achiral CDBA-OC12 which can also adopt a windmill-like conformation, even though the latter exists in large excess. This enantioselective pseudo-auto-adsorption turned out to be relevant to several unexpected self-assembling behaviours of cDBAs including the reversal of induced 2D chirality as discussed below. 


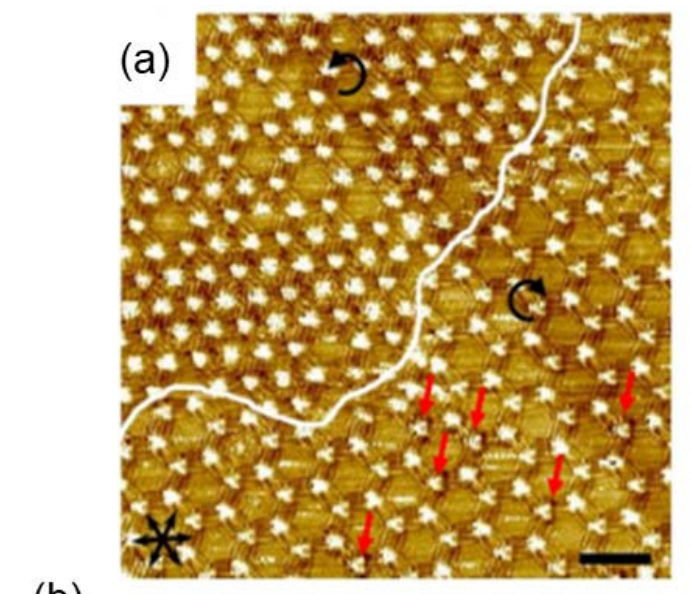

(b)

\section{cDBA-OC12(S)@CCW > cDBA-OC12(S)@CW}

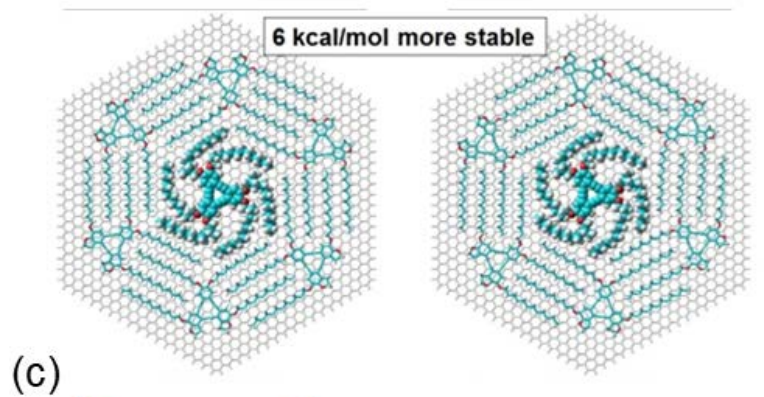

(c)

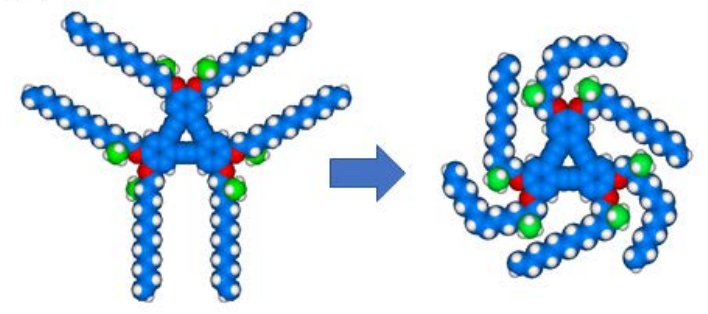

Fig. 9 (a) STM image of a premixed solution containing $30 \mathrm{~mol} \%$ CDBA-OC12(S) and 70 mol\% DBA-OC12. The white line marks the boundary between a CCW domain (top left) and CW domain (bottom right). The scale bar measures $5 \mathrm{~nm}$. The red arrows indicate CDBAOC12(S) molecules incorporated in the network of DBA-OC12. (b) Hexamer models of CCW (left) and CW (right) patterns of DBA-OC12 on a frozen graphene flake containing a pseudo-auto-adsorbed CDBAOC12(S) molecule adopting a windmill conformation in the pores. (c) Schematic for conversion from straight chain to windmill-like conformations of CDBA-OC12(S). Reproduced from Ref. 24 and 59 with permission from The Chemical Society of Japan and The Royal Society of Chemistry, respectively.

Annealing experiment carried out with the $10 \mathrm{~mol} \%$ cDBAOC12(S)/DBA-OC12 system under high concentration conditions $\left(2.5 \times 10^{-6} \mathrm{M}\right)$ resulted in a striking result of chirality reversal. ${ }^{54} \mathrm{At}$ room temperature the surface was covered by small domains, $81 \%$ of which consisted of CW pattern (Fig. 10a). On the contrary, upon annealing at $80^{\circ} \mathrm{C}$ the original supramolecular chirality was inverted, since a homochiral network of $\mathrm{CCW}$ pattern was formed accompanied by domain coalescence (Fig. 10b). This is in contrast to the result of annealing experiment at lower concentration $\left(5.0 \times 10^{-}\right.$ $7 \mathrm{M})$ described above, which resulted in the formation of large domain of the same handedness as that before annealing. Similar to the results of annealing at lower concentration, cDBA molecules were not observed in either the network or porous space, revealing the chiral memory effect. At intermediate concentrations, the system showed varying degree of chiral induction after annealing (Fig. 10c). By using an antipodal mixture of CDBA-OC12(R)/DBAOC12, CW homochiral surface was solely observed after annealing, confirming the reversal of the majority handedness. Undoubtedly, the chirality reversal is caused by preferential pseudo-autoadsorption of CDBA in its windmill-like conformation in the CCW porous network of achiral DBA, which drives the antipodal domains out at the boundaries where the adsorbed molecules are mobile upon thermal treatment.
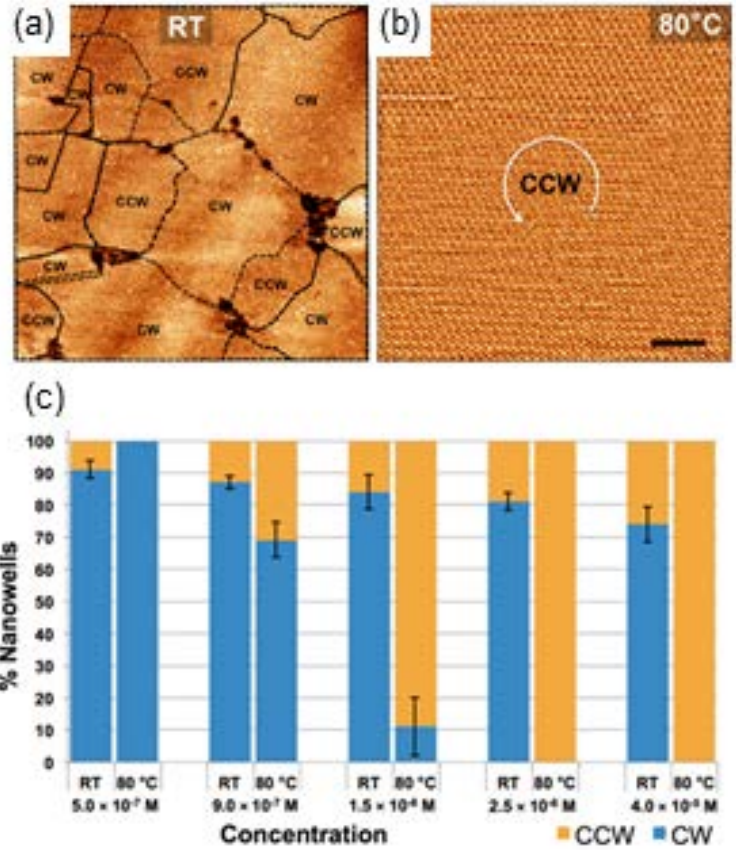

Fig. 10 (a) STM image of DBA-OC12 containing $10 \mathrm{~mol} \%$ cDBAOC12(S) at the PO/HOPG interface at room temperature $(C=2.5 \times$ $10^{-6} \mathrm{M}$ ). The surface consists of $81 \% \mathrm{CW}$ and $19 \% \mathrm{CCW}$ patterns. (b) STM image of the same solution after annealing at $80{ }^{\circ} \mathrm{C}$ (measurement at room temperature). The surface is covered solely by the CCW domain. (c) Histograms of concentration-dependent chirality induction observed at room temperature and after annealing at $80{ }^{\circ} \mathrm{C}$. The orange and blue bars indicate CCW and CW domains, respectively. Reproduced from Ref. 54 with permission from Springer Nature.

The different behaviour of CDBA-OC12(S) as a chiral inducer depending on the concentration and temperature is explained as follows. At low concentration, they are incorporated in the $\mathrm{CW}$ network of DBA-OC12 as sergeants at room temperature to define the chirality of the domain (Fig. 7). Upon annealing the handedness of the system is amplified through a ripening process. On the other hand, at high concentration, excess CDBA-OC12(S) molecules occupy 
CCW porous space preferentially as guests to form host-guest complexes (Fig. 9b). Upon annealing, the more stable CCW domains evolve through a ripening process to amplify the CCW patterns, from which cDBA molecules are finally desorbed leaving the memory of the homochiral network structure. It is also worthy of mentioning that the reversal of chirality was not observed when the experiment was done at the TCB/graphite interface. Using a $9: 1$ mixture of DBAOC12 and CDBA-OC12(S) $\left(C=1.0 \times 10^{-3} \mathrm{M}\right)$, only very weak preference of $\mathrm{CW}$ pattern was observed at room temperature. By contrast, the surface was covered exclusively with the same CW pattern after annealing accompanied by the disappearance of domain boundaries. Thus, at the interface of TCB and graphite, as CDBA-OC12(S) does not serve as a good guest to invert supramolecular chirality through the supramolecular host-guest pathway because of good solvating ability of TCB, it does work as an inducer of the sergeants-and-soldiers mechanism.

In the supramolecular host-guest mechanisms of chirality induction, the size matching between the porous space of the host network and the guest inducer molecule seems to be of critical importance. However, this assumption turned out to be not the case. In this respect, the effect of the network size on the efficiency of chirality induction through the supramolecular host-guest pathway was examined using a series of achiral DBA-OCn-evens smaller than CDBA-OC12 $(n=6,8,10)$ and larger than CDBA-OC12(S) $(n=14,16)$ as host components and CDBA-OC12(S) as a common chirality inducer at the $\mathrm{PO} /$ graphite interface. ${ }^{60}$ To prevent phase separation of domains formed by an achiral DBA and the CDBA and to assure avoiding incorporation of the CDBA in the network as a sergeant molecule, STM sample solutions were prepared by sequential deposition of an achiral DBA followed by (typically excess) cDBAOC12(S). After sequential deposition at room temperature, the formation of CW and CCW networks of the achiral DBA in equal proportions was confirmed. In the case of DBA-OC10, CDBA-OC12(S) was observed preferentially in the CCW pattern of the porous space when a large excess of the guest was used. After annealing, exclusive formation of CCW pattern was observed through chirality induction by the supramolecular host-guest pathway. On the other hand, the CDBA was not observed in the respective mixtures with DBA-OC6 and DBA-OC8; annealing resulted in domain ripening without noticeable chirality induction. Apparently, the porous space formed by these DBAs is too small for CDBA-OC12(S). In contrast, in the cases of large DBA-OC14 and DBA-OC16, preferential guest adsorption in CCW type porous space was observed. After annealing, the surface was covered solely with CCW pattern, indicating that the chirality induction through the supramolecular host-guest mechanism does not require precise matching between the host matrix and chiral guest. Indeed, only small stability differences are estimated, in particular for DBA-OC16, by the MM simulations for the host-guest complexes using hexamer models like those in Fig. 9b. These results nicely demonstrate how efficiently a small stability difference in a host-guest complex level is amplified to grow the thermodynamically more stable domain through the $2 \mathrm{D}$ crystal ripening process.
Another unexpected outcome resulting from the windmill-like conformation of CDBAs is the fact that they show remarkably higher preference to form porous network compared to achiral DBAs. ${ }^{61}$ For achiral DBAs at the TCB/graphite interface, the porous network is promoted at low concentration, while at higher concentration the densely packed linear structure is favoured. At the PO/graphite interface too DBA-OC12 forms the porous structure only at concentration lower than $1 \times 10^{-5} \mathrm{M}$. In contrast, cDBA-OC12(S) showed the porous structure independent on the concentration, covering more than $90 \%$ of the surface area in the concentration range of $2.5 \times 10^{-6}$ to $1.3 \times 10^{-3} \mathrm{M}$. The high preference of cDBAOC12(S) to form the porous network at the PO/graphite interface is ascribed to pseudo-auto-adsorption in the network pore, rendering the porous space no more porous. Due to the increased surface density, this pattern is stabilised enough to compete with linear pattern formation. At the TCB/graphite interface, such preference for the porous pattern was not observed, since pseudo-autoadsorption of cDBA was not preferred under this condition as described above.

\section{Stereospecific epitaxial growth}

Since we gained insight into control of the 2D chirality of SAMNs formed by DBAs at the monolayer level, we extended our attention to stereochemical control in the vertical dimension. Stereocontrolled epitaxial growth of SAMNs orthogonal to the surface is an interesting topic in view of its relevance to optoelectronic performance of surface-supported metal-organic frameworks (SURMOFs) ${ }^{62}$ and relationship between chiral molecular packing ${ }^{6}$ in $3 D$ and $2 D$ among others. However, only a few such studies have been reported for densely packed double layers grown using chiral molecules bearing twisted or helical backbones such as rubrene ${ }^{63}$ and helices ${ }^{64,65}$ under UHV condition and alkyl-substituted amino acid derivatives at the liquid/solid interface, ${ }^{66}$ in contrast to much more abundant studies on self-assembled bi- and multilayer formation without specific registry through $\pi-\pi$ stacking or dipolar interactions. ${ }^{67-71}$ For porous bilayer networks grown by self-assembly of acyclic building blocks, chirality did not take part, since only achiral bilayers were known. ${ }^{72,73}$ In this connection, to shed light on the possibility of stereocontrolled epitaxial growth of porous SAMNs, we designed CDBA-OC6-OH(S) and its enantiomer CDBA-OC6-OH(R) (Chart 1 ) bearing hydroxy groups at the stereogenic centres. ${ }^{74}$ Key features of the design (Fig. 11) are (1) the $C_{6}$ alkoxy chains to promote formation of the porous supramolecular structure, as we already revealed previously for DBA-OC6, ${ }^{75}$ with pores which are small enough to block autoadsorption, ${ }^{60}$ (2) the hydroxy groups introduced at the stereogenic centres which define $\mathrm{CW}$ and $\mathrm{CCW}$ patterns of the porous networks through (-) and (+) interdigitation modes of the alkoxy groups, respectively, similar to the chiral DBAs with methyl groups at the stereocentres, (3) the hydroxy groups, three of which should orient away from the surface when adsorbed on the surface as in the case of CDBA-OC12(S) (Fig. 6) and form interlayer hydrogen bonds to build up a bilayer (or multilayer) structure and (4) the hope for the stereospecific formation of one of possible structures of the bilayer through maximizing interlayer interactions. It should be pointed out 
that there exist two modes of stereospecificity, one with respect to intrinsic chirality of CDBA and the other supramolecular chirality of the network. The former specificity refers to the preferential bilayer formation between cDBAs of the same (e. g. $R$ over $R$ ) or opposite (e. g. $R$ over $S$ ) handedness when a racemic mixture is used, and the latter refers to the selection between the same (e. g. CW over CW) or opposite (e. g. CCW over CW) handedness of the network. Additionally, the most critical technical matter in this study was the bias voltage-dependent STM imaging which made it possible to discriminate the DBA molecules in the top layers from those in the layer underneath.

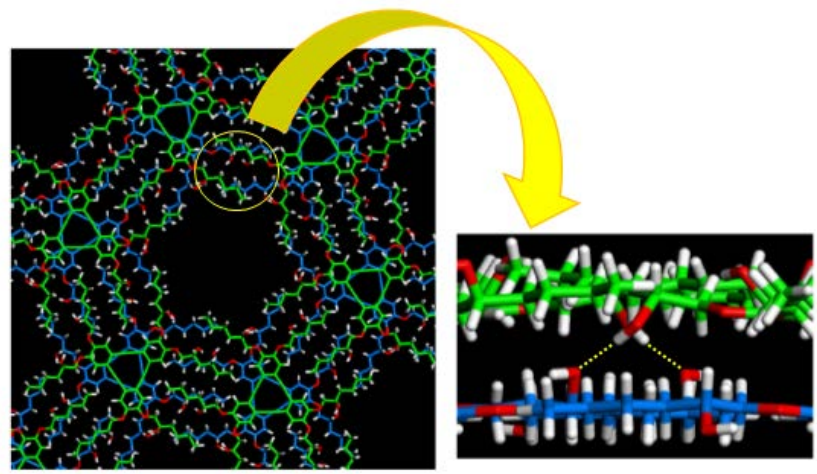

Fig. 11 Part of MM-optimized molecular model of the porous double layer formed by top layer CDBAOC6-OH(R) (green framework) on underlaying CDBAOC6-OH(R) (blue framework) through interlayer van der Waals and hydrogen bonding interactions. Two of possible hydrogen bonding interactions among others are shown by dotted yellow lines in the side view (right).

At relatively low concentration of $2.3 \times 10^{-6} \mathrm{M}$ at the $\mathrm{TCB} /$ graphite interface, homochiral monolayer of either $\mathrm{CW}$ or $\mathrm{CCW}$ pattern was formed from CDBA-OC6-OH(S) or CDBA-OC6-OH(R), respectively, exhibiting the same selectivity for the supramolecular chirality as those observed for CDBAs with methyl groups at the stereogenic centres. This indicates that the same mechanism due to the steric hindrance of the hydroxy groups plays a role to define the monolayer chirality. Deposition of a racemic mixture results in the formation of phase separated enantiomorphous domains with equal surface coverage. Upon slightly increasing the concentration to $2.6 \times$ $10^{-6} \mathrm{M}$, pairs of bright triangle images started to appear as shown in Fig. 12a. A line profile (Fig. 12e) shows that the height of the bright DBA molecule is almost twice as high as the majority DBA molecules, indicating that the bright triangles are due to DBA molecules in the second layer. We also notice that the neighbouring bright triangles are facing to each other in contrast to those in the first layer which are shifted to each other to form a chiral network (Fig. 12b-d). The different appearance between the top layer molecules and those in the layer underneath is indicative of different orientations of the DBA molecules in top and bottom layers. The different alignment of the DBA molecules in the top and bottom layers becomes more prominent in the images at higher concentration as described below.

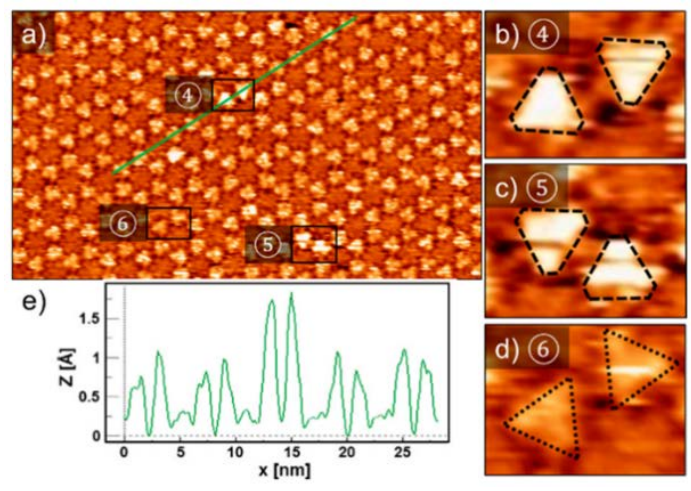

Fig. 12 (a) High-resolution STM image of CDBA-OC6-OH(S) at the $\mathrm{TCB} /$ graphite interface at $\mathrm{C}=2.6 \times 10^{-6} \mathrm{M}$. (b-d) Digital magnification of the image shown in (a). Bright triangles in (b) and (c) appear to face each other as opposed to being shifted for those in (d). (e) Line profile across a pair of bright triangular images as indicated by the green line in a. Reproduced with permission from Ref. 74. Copyright 2020 American Chemical Society.

By further increasing the concentration, the image contrast became dependent on the bias voltage. The two sequential images for CDBA-OC6-OH(R) $\left(1 \times 10^{-3} \mathrm{M}\right)$ taken $2 \mathrm{~min}$ apart at different bias voltages are shown in Fig. 13a and 13b. Fig. 13a which was recorded at the bias voltage $\left(V_{\text {bias }}\right)$ of $-0.5 \mathrm{~V}$ contains narrow domain boundaries as indicated by white dashed lines and is consistent with a SAMN of CDBA. By contrast, Fig. $13 \mathrm{~b}$ which was recorded at $V_{\text {bias }}$ of $-1.2 \mathrm{~V}$ contains some dark trenches that appear to coincide with the white lines in Fig. 13a. Based on the different appearance of the domain boundaries and the height profile, we attribute the image with dark trenches (Fig. 13b) observed at the more negative sample bias to the top layer of a multilayer structure. The bias voltage can be switched reversibly to image multiple and single layers during scanning (Fig. 13c). When the voltage was switched from -1.2 (larger negative bias) to $-0.5 \mathrm{~V}$ (smaller negative bias), the top half appeared brighter than the bottom half, corresponding to the imaging of a multiple layer and a monolayer of the same domain. As to the reason for the different appearance, we assume that upon decreasing the absolute value of the voltage the STM tip likely penetrates through the double layer to image the bottom layer since the tip-to-substrate distance decreases. 

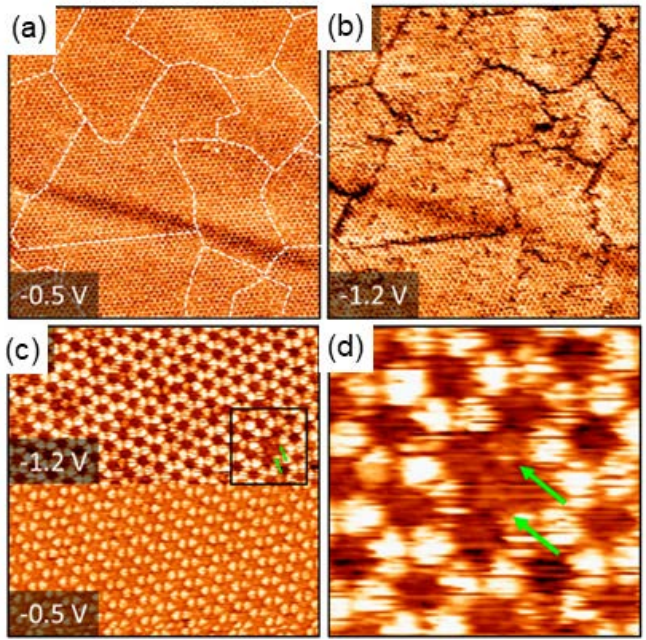

Fig. $13(a, b)$ Sequential STM images of CDBA-OC6-OH(S) at $\mathrm{TCB} /$ graphite interface $\left(1 \times 10^{-3} \mathrm{M}\right)$ at a bias voltage of $-0.5 \mathrm{~V}(\mathrm{a})$ and $-1.2 \mathrm{~V}(\mathrm{~b})$, respectively. White dashed lines in (a) indicate domain borders. (c) STM image of CDBA-OC6-OH(S) in which the voltage was increased from $-1.2 \mathrm{~V}$ to $-0.5 \mathrm{~V}$ in the middle of the image from top to bottom. (d) Magnification of the area indicated by black square in (c). In the defects of the top layer, molecules in the lower layer (marked by green arrows) are imaged darker than the surrounding molecules. Reproduced with permission from Ref. 74. Copyright 2020 American Chemical Society.

With this powerful tool in hand, which made it possible not only to discriminate monolayers and multilayers but also to assign the supramolecular chirality (CW or CCW pattern) of the bottom layer network, we investigated diastereospecificity in the multilayer formation. With respect to intrinsic chirality of the building blocks forming multilayers, the following experiment was performed. A solution of racemic mixture in TCB was placed in a liquid cell with graphite substrate. After network formation was confirmed, an excess amount of one of the enantiomers, CDBA-OC6-OH(R) was added to the liquid cell. STM imaging at a small negative bias of -0.6 $\checkmark$ revealed that the $C W$ and $C C W$ domains remained separated in the first layer (Fig. 14a). By decreasing the bias voltage to $-1.2 \mathrm{~V}$, a multilayer was observed exclusively at the CCW domain formed by CDBA-OC6-OH(R) (Fig. 14b), indicating that the top layer also consists of CDBA-OC6-OH(R). Similar results were obtained when excess CDBA-OC6-OH(S) was added with exclusive multilayer formation in the CW domain.

In addition, by switching the bias voltage during imaging, we were able to monitor each layer separately (Fig. 14c). Indeed, these measuring conditions confirm that in the presence of excess CDBA$\mathbf{O C 6}-\mathbf{O H}(R)$, bilayer formation is only observed on top of the CCW bottom layer domains which consists of the same enantiomer. These experiments clearly show that the multilayers are formed diastereospecifically on top of the same enantiomer of CDBA.
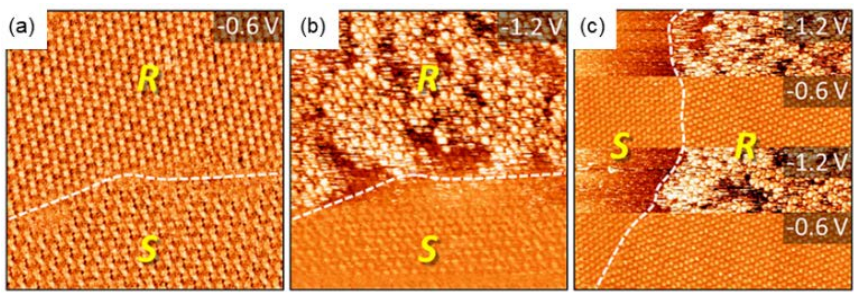

Fig. $14(a-c)$ STM images acquired by sequential addition of a 1:1 mixture of CDBA-OC6-OH(S) and CDBA-OC6-OH(R) (concentration of each enantiomer $\left.=3 \times 10^{-4} \mathrm{M}, 20 \mu \mathrm{L}\right)$, followed by addition of CDBAOC6-OH $(R)\left(1.2 \times 10^{-3} \mathrm{M}, 20 \mu \mathrm{L}\right)$ at the TCB/graphite interface. White dashed lines indicate domain boundaries. ( $a$ and b) Sequential images of the same location. (a) Image acquired at $-0.6 \mathrm{~V}$ showing the top and bottom domains are CDBA-OC6-OH(R) and CDBA-OC6$\mathrm{OH}(\mathrm{S})$, respectively. (b) Image at $-1.2 \mathrm{~V}$ showing the double-layer is formed only on top of the domain of CDBA-OC6-OH(R). (c) STM image obtained by modulating the bias voltage during the scanning process as indicated in the figure. Reproduced with permission from Ref. 74. Copyright 2020 American Chemical Society.

Knowing the diastereospecific multilayer formation with respect to the intrinsic chirality of CDBAs, we next investigated diastereospecificity at the supramolecular level, namely the relationship between $\mathrm{CW}$ or CCW patterns of the first and second layers. Fig. 15a shows an STM image of CDBA-OC6-OH(S) $\left(1 \times 10^{-3} \mathrm{M}\right)$ at the TCB/graphite interface. The triangular features, which are highlighted by the black dotted lines, form a pattern with $p 6 m$ plane group symmetry ${ }^{76}$ in which the neighbouring triangular features are facing, in contrast to the chiral $p 6$ symmetry ${ }^{75}$ of the monolayer (Fig. $15 \mathrm{a}$, inset) in which triangles are shifted. The same characteristic appearance of bright triangles was observed at lower concentration (Fig. 12). We attributed the appearance of this achiral feature to an offset of shifts between overlapping antipodal triangles, as shown in Fig.15b. Accordingly, we deduce that the second layer adopts an antipodal supramolecular chirality, i.e., $\mathrm{CCW}$ on top of $\mathrm{CW}$ in the case of CDBA-OC6-OH(S) and CW on CCW for CDBA-OC6-OH(R). Unfortunately, we are unable to rationalise the observed diastereoselectivity by using MM simulations, since the observed selectivity is likely of kinetic origin. The experiment with respect to the selective formation of multilayer on the domain formed by CDBA of the same chirality (Fig. 13) already suggests that the multilayer formation is a kinetically trapped metastable state. However, the experimental results demonstrate that a high level of selectivity in multilayer formation can be attained by a combination of multiple noncovalent interactions.
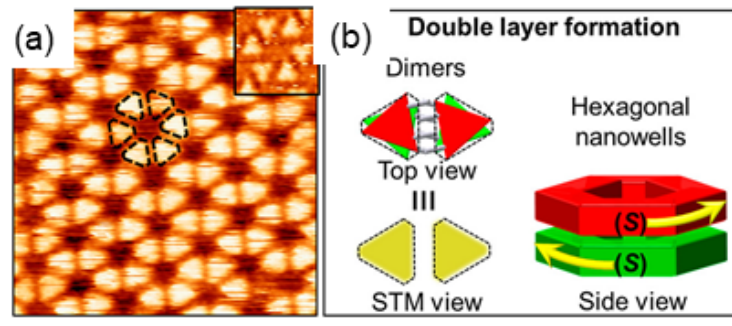
Fig. 15 (a) STM image of a multilayer of CDBA-OC6-OH(S) $\left(V_{\text {bias }}=-1.4\right.$ V) showing the apparent orientations of the triangle DBA cores of the multilayer by black dashed triangles. (Inset) Monolayer self-assembly of CDBA-OC6-OH(S) for comparison between the plane groups chiral p6 (double layer) to achiral $\mathrm{p} 6 \mathrm{~m}$ (monolayer). (b) Proposed multilayer growth model where the bottom layer and the second layer have opposite supramolecular chirality. At the dimer level for CDBA-OC6-OH(S), the bottom layer (green) adopts CW hexagonal pattern via the (-) interdigitation mode, while the second layer (red) adopts CCW pattern via the (+) interdigitation mode. Red and green triangle cores are right on top of each other but rotated. The superimposed image outlined by the dotted triangles will result in STM contrast of facing triangles. Reproduced with permission from Ref. 74. Copyright 2020 American Chemical Society.

\section{Chirality transfer using porous networks as chiral template for surface grafting}

The final section of this article describes our recent attempt to use chiral SAMNs as templates of chemical functionalisation of flat carbon surfaces such as graphene and graphite to create a topologically chiral surface. Since we knew that DBA forms SAMNs of domain-specific chirality whose unit cell angles are tilted with respect to the graphite lattice (step 1: Fig. 16), we envisioned that if chemical functionalisation takes place at the centre of the porous space (step 2: shown by red dots), the resulting modified graphite after removal of the template (step 3 ) should also have domain specific chirality because of the right- or left-handed direction of the tilt angle of the templates. By using a homochiral CDBA as a template, topologically homochiral modified graphite would be created, ${ }^{77}$ similar to the situation of chiral nanotubes which also possess chirality of topological origin. ${ }^{78}$ Though atomic-level positional control in chemical modification of flat graphitic materials has attracted enormous interest mainly in connection with band gap control of graphene, ${ }^{79-81}$ to our knowledge, there exists no report on chirality control in functionalisation. It should also be pointed out that the topologically chiral graphitic materials are different from those bearing chiral functional groups randomly grafted on the surface. ${ }^{7-11}$

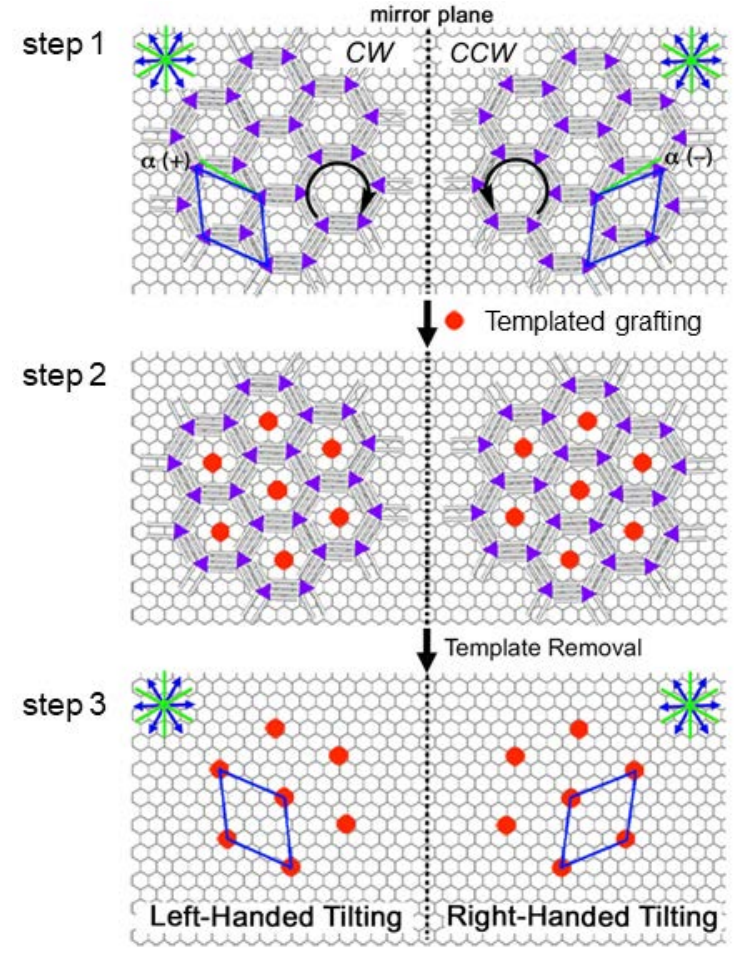

Fig. 16 Schematic drawing of the porous template-guided hexagonal grafting on top of a honeycomb graphene/graphite lattice. Blue and light green arrows are the main symmetry axes of graphene/graphite and their normal, respectively. The purple triangles and faint lines connecting them represent the DBA cores and alkyl chains, respectively. Blue rhombi in the top and bottom panels are the unit cells of the DBA networks and those of the grafted species assuming grafting in the centre of the pores, respectively. The supramolecular template network forms domain specific mirror images of clockwise (CW, left) and counterclockwise (CCW, right) patterns. Though the resulting pattern of the grafted molecules (red dots) is achiral by itself (plane group $p 6 \mathrm{~m}$ ), the tiling between the SAMN unit cell vectors and the directions normal to the main symmetry axes of graphite renders the functionalized surface topologically chiral. Reproduced with permission from Ref. 77. Copyright 2020 American Chemical Society.

To realise the above prospect, a few problems had to be solved. The first problem was the method of covalent grafting giving graphitic surface of high grafting density. We selected C-C bond forming reaction of an aryl radical generated by electrochemical reduction of the corresponding aryldiazonium ion, not only because it is a well-known method ${ }^{82-84}$ but also because we revealed that aryl radicals protected by electron-donating groups at the meta- and para-positions, such as 3,5-di-tert-butyl and 3,4,5-trimethoxy derivatives, gave monolayer grafting with high density (Fig. 17a). ${ }^{85.86}$ On the other hand, aryl radicals with electron-withdrawing groups resulted in low-density grafting and those without protecting groups formed multi-layers by further radical attack to the grafted aryl groups. The grafting density can be conveniently confirmed by STM and Raman spectroscopy. The second critical issue was the efficiency of SAMNs as template for grafting by aryl radicals. Even though 
chemically modified aryldiazonium ions had been used for siteselective grafting of graphite, ${ }^{87-89}$ the use of SAMNs as a molecularlevel template was unknown before we achieved width-controlled linear grafting of graphite and graphene using lamella type SAMNs of long straight-chain alkanes as templates. ${ }^{90}$ We used an electrochemical (EC) cell in which graphite or graphene was used as a working electrode which is usual for diazonium grafting. However, the key to the successful site-selective grafting was the formation of a phase-separated solution double layer, consisting of a very thin solution layer of the large $n$-alkane $\left(\mathrm{C}_{30} \mathrm{H}_{62}, \mathrm{C}_{40} \mathrm{H}_{82}\right.$ or $\left.\mathrm{C}_{50} \mathrm{H}_{102}\right)$ template in tetradecane solvent and an aqueous solution of the diazonium salt (Fig. 17b). By using this setup, covalent grafting was directed to the unit cell vertices where the alkane terminals meet (Fig. 17c), forming a quasi-linear alignment of aryl groups grafted on the graphite surface as shown in Fig. 17d. More importantly, the widths between the lines of the aryl groups as determined by 2D Fast Fourier Transform (2D-FFT) are $4.1 \mathrm{~nm}, 5.8 \mathrm{~nm}$ and $6.8 \mathrm{~nm}$ for $\mathrm{C}_{30} \mathrm{H}_{62}$, $\mathrm{C}_{40} \mathrm{H}_{82}$ and $\mathrm{C}_{50} \mathrm{H}_{102}$ templates, respectively, which virtually match the lamella widths of the alkane templates measured by STM $(4.0 \mathrm{~nm}$, $5.3 \mathrm{~nm}$ and $6.6 \mathrm{~nm}$, respectively), indicating clearly that the alkane monolayers serve as templates. The template layer was removed by washing with hot chloroform leaving the grafted aryl groups intact. By contrast, the use of dried lamellar type monolayer of pentacontane on graphite merely blocked the grafting of the graphite surface..$^{91}$ These results suggest that the presence of the thin layer of tetradecane solution allows lateral displacement of the alkane terminal groups creating temporal space for radical attack (Fig. 17c). Quasi-linear grafting with a periodicity of $4.2 \mathrm{~nm}$ was also possible on single layer graphene on copper using a triacontane mask, although the pattern transfer fidelity was weaker as revealed by the bent rows and defects in the STM image and the weak intensities of the 2D-FFT image.
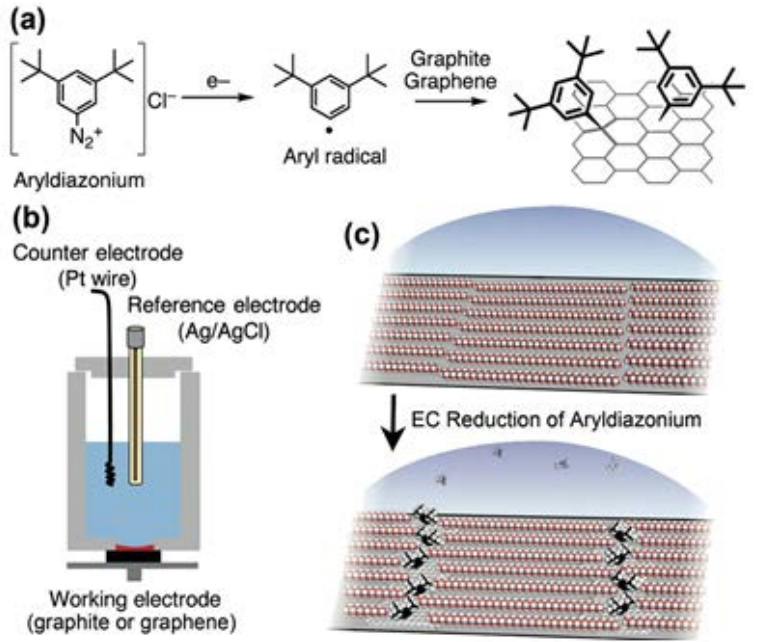

(d)

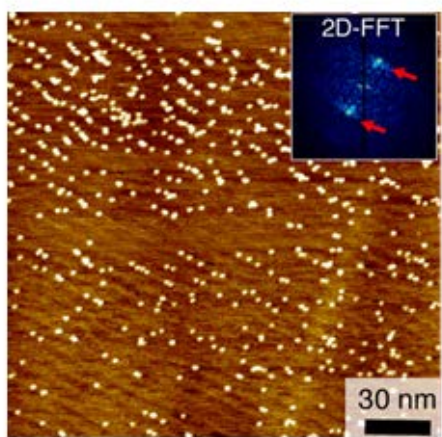

Fig. 17 (a) Schematic representation for chemical covalent functionalization of graphitic surfaces by an aryl radical generated from 3,5-di-tert-butylbenzenediazonium chloride. (b) An illustration for an EC cell contains a solution double layer consisting of an aqueous phase containing diazonium molecules (light blue) and a thin organic layer of template solution (red), in which lamellar type SAMN of a large alkane is formed at the interface of solution and graphite electrode. (c) An illustration for linear grafting using a SAMN of pentacontane template as an example. (d) STM image of the electrochemical grafting of graphite with 3,5-di-tertbutylbenzenediazonium chloride showing linearly aligned rows of bright dots due to the covalently bound aryl groups. Inset shows a 2D-FFT indicating a one-dimensional orientational control highlighted with red arrows. Reproduced with permission from Ref. 90. Copyright 2020 American Chemical Society.

Now the stage was set for 2D pattern transfer using porous SAMNs with domain-specific supramolecular chirality as templates. For this purpose, we selected DBA-OCns of $n=4,6$ and 8 , which were expected to form a porous space comparable with a grafted aryl group. Namely, the size of their porous space increases with increasing alkoxy chain length. The shortest pore-to-pore distances are 2.4, 2.8 and $3.1 \mathrm{~nm}$, respectively. The same EC setup with a phase-separated solution double layer as above was used except for 3,4,5-trimethoxyphenyldiazonium chloride as the radical precursor and PO as the solvent of the thin solution layer. An STM image obtained after grafting using DBA-OC8 as a template molecule is shown in Fig 18a,b. It shows the trimethoxyphenyl units as bright 
features together with dim features which are assigned to the DBA cores of the network. Though the network structure is considerably destroyed, resulting in random grafting at bare surface, a 2D-FFT of the STM image (inset Fig 18a) clearly shows the hexagonal periodicity of the grafted aryl groups with a mean periodicity of $3.0 \mathrm{~nm}$, which matches the pore-to-pore distance of the DBA-OC8 molecular network. Grafting using the DBA-OC6 template led to hexagonal periodicity of $2.7 \mathrm{~nm}$, which also agrees with the pore-to-pore distance of DBA-0C6 molecular network. However, with the DBAOC4 template, only weak hexagonal periodicity of $2.3 \mathrm{~nm}$ was observed in the STM images and 2D-FFT analysis, indicating the guiding effect is limited due to the too small pore size. These results support our hypothesis for the pattern transfer from the SAMNs of DBAs to the grafted location of aryl groups on graphite. Removing the physisorbed SAMN template is possible by washing with hot toluene, again leaving the grafted aryl groups intact.
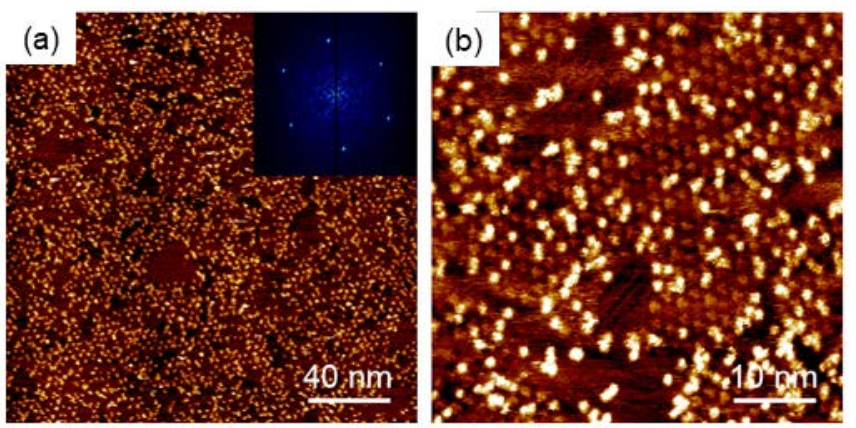

Fig. 18 STM images of functionalized graphite surface after DBA-OC8templated grafting in 1-phenyloctane. (a) Large-area image. The inset corresponds to the 2D-FFT image. Six bright spots in the 2D-FFT image confirm the hexagonal periodicity. (b) Small-area image Reproduced with permission from Ref. 77. Copyright 2020 American Chemical Society.

To prove chirality transfer, we relied on the offset angles between the unit cell vector of SAMNs and the normal to a main symmetry axis of graphite (angle $\alpha$ ). For the network formed by DBAOC6, the absolute value of the angle $\alpha$ is $\pm 6.3^{\circ}$ (Table 1 ). The angle between the unit cell vectors of the $\mathrm{CW}$ and CCW structures $(B)$ is determined to be $13^{\circ}$ directly from the 2D FFT analysis of an STM image which includes both $\mathrm{CW}$ and CCW domains. We hypothesized that this chirality information will be transferred to the position of the covalently attached aryl groups as described above (Fig. 16). Using the DBA-OC6 template, we were able to observe two different orientations in the hexagonal alignment of the grafted aryl groups in STM images of the functionalized graphite surface (Fig. 19a). The boundary between the domains is highlighted by a blue line. The corresponding 2D-FFT image (Fig. 19b) shows two sets of hexagonal periodicities corresponding to each domain. The angle between the two periodicities $\left(14^{\circ}\right)$ agrees with the angle $B$ of the template DBAOC6 network. Moreover, the offset $\left( \pm 6.5^{\circ}\right)$ between the unit cell vector of the grafted nanopattern and the normal to a main symmetry axis of graphite also agrees with that for the DBA-OC6 SAMN $\left( \pm 6 \cdot 3^{\circ}\right)$. As expected, domains of "positive" and "negative" offsets appear with almost equal frequency. These results clearly support our hypothesis that the handedness of the hexagonal network is transferred to the location of the covalently attached aryl units.

Finally, to create a globally homochiral grafted pattern using a supramolecular template layers of unique handedness, we synthesized cDBA-OC6(S)-OC6, a chiral template molecule having three chiral $C_{6}$ alkoxy chains with methyl groups attached to stereogenic centres and three achiral $C_{6}$ chains. Similar to cDBAOC12(S)-0C12 described above, CDBA-OC6(S)-OC6 exclusively forms a CCW honeycomb structure at the TCB/graphite interface. We note that the offset angle $\alpha$ measures only negative values of $-6.7^{\circ}$, consistent with the homochirality of the network. Electrochemical grafting was performed under the double layer conditions using a solution of CDBA-OC6(S)-OC6 in TCB. STM observations revealed that individual aryl units arranged in a hexagonal manner with a hexagonal periodicity of $2.6 \mathrm{~nm}$ based on the 2D-FFT (Fig. 19c). Furthermore, the orientation of the grafted pattern with respect to the normal of the symmetry axes of graphite exhibits only negative value of $-6.8^{\circ}$, matching exactly that of CDBA-OC6(S)-OC6 SAMN. This indicates that the unique handedness of the template SAMN guided the location of grafting, rendering the covalently functionalized graphite surface topologically homochiral (Fig. 19d).
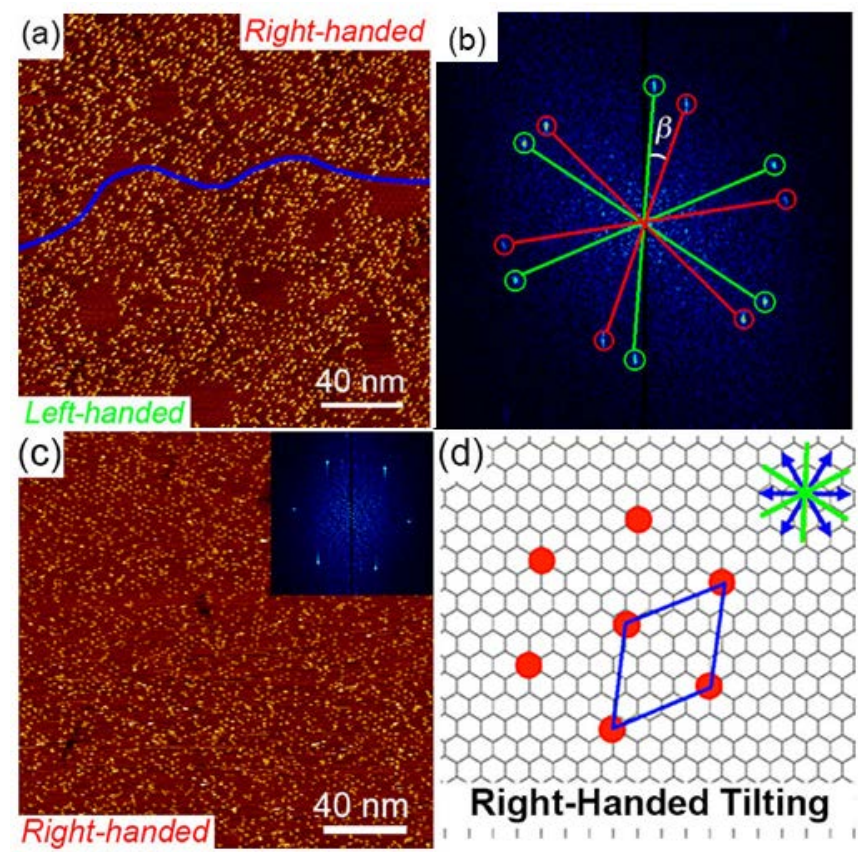

Fig. 19 (a) Large-area STM image of the functionalized graphite surface around the domain boundary after DBA-OC6-templated grafting in 1-phenyloctane. Blue line indicates a boundary of hexagonally aligned bright features with different orientations. (b) 2D-FFT images of image in (a). Two sets of six bright spots marked by green and red circles are derived from the bottom and top domains of (a). The mean angle ( $b$ ) between the green and red lines connecting the spots and the centre of the image is $14 \pm 2^{\circ}$. (c) Largearea STM image of the functionalized graphite surface after CDBAOC6(S)-OC6-templated grafting in TCB. The inset corresponds to the 
2D-FFT image. (d) Schematic drawing of the chiral-positioncontrolled functionalized surface. The red dots correspond to grafted aryl units. Blue arrows and light green lines are the main symmetry axes and their normals of a graphite substrate, respectively. Reproduced with permission from Ref. 77. Copyright 2020 American Chemical Society.

\section{Conclusions and outlook}

We have demonstrated several aspects of supramolecular chirality control related to the porous SAMNs formed by DBA derivatives. Firstly, we found that 2D chirality induction took place efficiently using a small amount of CDBA-OC12(S), a chiral inducer structurally similar to achiral DBA-OC12. Domain-specific chiral but globally achiral SAMN were formed through a classic sergeants-and-soldiers mechanism, in which the chiral inducer was incorporated into the supramolecular network. Next, through careful inspections of the $2 \mathrm{D}$ chirality of the SAMNs, we realized that chirality reversal occurred when the solute concentration was relatively high. Based on STM experiments and MM simulations, we concluded that the reversal of chirality was driven by a hitherto unknown "supramolecular hostguest" mechanism, in which the chiral inducer molecule was selectively entrapped in the porous space of specific handedness as a guest by changing its global shape to a windmill-like conformation. We also extended the dimension of stereochemical control in selfassembly from 2D to 3D by demonstrating diastereospecific bilayer formation through interlayer hydrogen bonding of hydroxycontaining chiral DBA, CDBA-OC6(S)-OH, both at the single molecule level as well as the supramolecular orientation between the top and bottom layers. These results, some of which arose from the unexpected behaviour of molecular building blocks, suggest the prospect to create more complex and sophisticated self-assemblies on the surfaces. This may be achieved by designing building blocks through, for example, balancing the flexible and rigid parts of molecular building blocks and using multiple orthogonal noncovalent forces for them to assemble in a specific manner under certain external conditions (such as concentration or temperature). In this respect, implementation of the concept of self-sorting 92,93 and integrative self-sorting, ${ }^{94,95}$ which have been topics of active research in solution self-assembly, would open a new direction in 2D selfassembly. Our most recent results on the formation of a highly hierarchical SAMN, yet not related to chirality, support that this must be the case. ${ }^{96}$

Even though stereocontrolled epitaxial growth of SAMNs orthogonal to the surface is a challenging topic, only few scattered investigations have been performed, in spite of its potential relevance to SURMOFs. ${ }^{62}$ This is probably not only because it may seem unsurmountable to control the intermolecular interactions in multiple dimensions to build up a stereochemically defined architecture. It is also due to the difficulty to determine the stereochemical relationship in vertical direction using the available microscopic techniques particularly when the thickness of the multilayer increases. ${ }^{70}$ Related to this, our successful observation of the bilayered porous SAMNs formed by CDBA-OC6-OH(S) and CDBA$\mathbf{O C 6}-\mathrm{OH}(R)$ by the use of orthogonal noncovalent forces, van der
Waals and hydrogen bonding interactions, shed light on protocols for stereocontrolled epitaxial growth of porous SAMNs. Moreover, bias voltage-dependent selective observation of the upper and lower layers provides useful information for this technical challenge.

We demonstrated, for the first time, that porous SAMNs formed by DBA-OC6 and DBA-OC8 act as templates for the covalent functionalization of graphite by an aryl radical generated from the corresponding aryldiazonium salt by EC reduction, though the fidelity of grafting was far from perfect. The masks can be removed by washing with common organic solvents. Moreover, we confirmed that the orientation of the porous template network with respect to the graphite symmetry axes was transferred to the alignment of the grafted aryl groups along nonsymmetry axes of graphite. By using homochiral SAMNs formed by CDBA-OC6(S)-OC6, a homochiral functionalised carbon surface is created which is a hitherto unknown topologically chiral graphite material. Though improvement of pattern transfer fidelity is necessary, the advantage of the present approach is the spatial control in the few-nanometer regime, tunable by simply changing the size of the alkoxy chains. As such it is highly relevant for the positionally controlled functionalization of graphene, ${ }^{97}$ an approach which is of interest in view of band gap control of graphene. ${ }^{79-81}$ The production of homochiral graphite surfaces or graphene materials would open various possibilities in carbon materials science, such as chirality dependent band gap control of graphene, ${ }^{12}$ chiroptical sensors and other potential applications. . $^{5-14,98}$

\section{Conflicts of interest}

There are no conflicts to declare.

\section{Acknowledgements}

This work is supported by JSPS KAKENHI (JP15H02164, JP17H04794, JP17K19130, JP19H04597, JP2OH02553), the Fund of Scientific Research Flanders (FWO), and KU Leuven-Internal Funds.

\section{Notes and references}

1 S. M. Barlow and R. Raval, Sur. Sci. Rep., 2003, 50, 201-341.

2 K.-H. Ernst, Z. Phys. Chem., 2009, 223, 37-51.

3 K.-H. Ernst, Phys. Status Solidi B, 2012, 249, 2057-2088.

4 J. A. A. W. Elemans, I. De Cat, H. Xu and S. De Feyter, Chem. Soc. Rev., 2009, 38, 722-736.

5 F. Zaera, Chem. Soc. Rev., 2017, 46, 7374-7398.

6 S. Dutta and A. J. Gellman, Chem. Soc. Rev., 2017, 46, 77877839

7 R. M. Hazen and D. S. Sholl, Nat. Mater., 2003, 2, 367-374.

8 X. Feng, B. Shen, B. Sun, J. Kim, X. Liu and M. Lee, Angew. Chem. Int. Ed., 2020, 59, 11355-11359.

9 D. Y. Murzin, P. Maäki-Arvela, E. Toukoniitty and T. Salmi, Catal. Rev., 2005, 47, 175-256.

10 M. Heitbaum, F. Glorius and I. Escher, Angew. Chem. Int. Ed., 2006, 45, 4732-4762.

11 T. Mallat, E. Orglmeister and A. Baiker, Chem. Rev., 2007, 107, 4863-4890.

12 X. Liu, Z. Zhang and W. Guo, small, 2013, 9, 1405-1410. 
13 J. X. Lian, A. Lherbier, L. J. Wang, J. C. Charlier, D. Beljonne and Y. Olivier, J. Phys. Chem. C, 2016, 120, 20024-20032.

14 S. J. Yoo and Q.-H. Park, Nanophotonics, 2019, 8, 249-261.

15 D. P. Goronzy, M. Ebrahimi, F. Rosei, Arramel, Y. Fang, S. De Feyter, S. L. Tait, C. Wang, P. H. Beton, A. T. S. Wee, P. S. Weiss and D. F. Perepichka, ACS Nano, 2018, 12, 7445-7481.

16 M. Forster, M. S. Dyer, M. Persson and R. Raval, J. Am. Chem. Soc., 2011, 133, 15992-16000.

17 A. G. Mark, M. Forster and R. Raval, ChemPhysChem, 2011, 12, $1474-1480$

18 T. Chen and D. Wang, L.-J. Wan, Natl. Sci. Rev., 2015, 2, 205 216.

19 K.-H. Ernst, Orig. Life Evol Biosph., 2010, 40, 41-50.

20 S. De Feyter and F. C. De Schryver, Chem. Soc. Rev., 2003, 32, 139-150.

21 J. A. A. W. Elemans, S. Lei and S. De Feyter, Angew. Chem. Int. Ed., 2009, 48, 7298-7332.

22 K. Tahara, S. Lei, J. Adisoejoso, S. De Feyter and Y. Tobe, Chem. Commun., 2010, 46, 8507-8525.

23 S. Lei, K. Tahara, J. Adisoejoso, T. Balandina, Y. Tobe and S. De Feyter, CrystEngComm, 2010, 12, 3369-3381.

24 Y. Tobe, K. Tahara and S. De Feyter, Bull. Chem. Soc. Jpn., 2016, 89, 1277-1306

25 K. Iritani, K. Tahara, S. De Feyter and Y. Tobe, Langmuir, 2017, 33, 4601-4618

26 J.-H. Kim, J. Jung, K. Tahara, Y. Tobe, Y. Kim and M. Kawai, J. Chem. Phys., 2014, 140, 074709.

27 J. Park, J.-H. Kim, S. Bak, K. Tahara, J. Jung, M. Kawai, Y. Tobe and Y. Kim, Angew. Chem. Int. Ed., 2019, 58, 9611-9618.

28 S. Kawai, V. Haapasilta, B. D. Lindner, K. Tahara, P. Spijker, J. A. Buitendijk, R. Pawlak, T. Meier, Y. Tobe, A. S. Foster and E. Meyer. Nat. Commun., 2016, 7, 12711

29 T. Balandina, K. Tahara, N. Sändig, M. O. Blunt, J. Adisoejoso, S. Lei, F. Zerbetto, Y. Tobe and S. De Feyter, ACS Nano, 2012 6, 8381-8389.

30 K. Schouteden, T. Ivanova, Z. Li, V. Iancu, K. Tahara, Y. Tobe, J. Adisoejoso, S. De Feyter, C. Van Haesendoncka and E. Janssensa, Chem. Commun., 2015, 51, 10917-10920.

31 S.Furukawa, H. Uji-i, K. Tahara, T. Ichikawa, M. Sonoda, F. C. De Schryver, Y. Tobe and S. De Feyter, J. Am. Chem. Soc., 2006, 128, 3502-3503.

32 K. Tahara, S. Furukawa, H. Uji-i, T. Uchino, T. Ichikawa, J. Zhang, W. Mamdouh, M. Sonoda, F. C. De Schryver, S. De Feyter and Y Tobe, J. Am. Chem. Soc., 2006, 128, 16613-16625.

33 S. Lei, K. Tahara, F. C. De Schryver, M. Van der Auweraer, Y. Tobe and S. De Feyter, Angew. Chem. Int. Ed., 2008, 47, 29642968.

34 M. O. Blunt, J. Adisoejoso, K. Tahara, K. Katayama, M. Van der Auweraer, Y. Tobe and S. De Feyter, J. Am. Chem. Soc., 2013, $135,12068-12075$.

35 H. Cao, K. Tahara, S. Itano, Y. Tobe and S. De Feyter, J. Phys. Chem. C, 2017, 121, 10430-10438.

36 S. Furukawa, K. Tahara, F. C. De Schryver, M. Van der Auweraer, Y. Tobe and S. De Feyter, Angew. Chem. Int. Ed. 2007, 46, 2831-2834.

37 S. Lei, K. Tahara, X. Feng, S. Furukawa, F. C. De Schryver, K. Müllen, Y. Tobe and S. De Feyter, J. Am. Chem. Soc., 2008, 130, 7119-7129.

38 K. Tahara, H. Yamaga, E. Ghijsens, K. Inukai, J. Adisoejoso, M. O. Blunt, S. De Feyter and Y. Tobe, Nat. Chem., 2011, 3, 714719.

39 E. Ghijsens, O. Ivasenko, K. Tahara, H. Yamaga, S. Itano, T. Balandina, Y. Tobe and S. De Feyter, ACS Nano, 2013, 7, 80318042.

40 H. Cao, I. Destoop, K. Tahara, Y. Tobe, K. S. Mali and S. De Feyter, J. Phys. Chem. C, 2016, 120, 17444-17453.

41 A. Minoia, I. Destoop, E. Ghijsens, S. De Feyter, K. Tahara, Y. Tobe and R. Lazzaroni, RSC Adv., 2015, 5, 6642-6646.
42 K. Tahara, A. Noguchi, R. Nakayama, E. Ghijsens, S. De Feyter and Y. Tobe, Angew. Chem. Int. Ed., 2019, 58, 7733-7738.

43 M. M. Green, N. C. Peterson, T. Sato, A. Teramoto, R. G. Cook and S. Lifson, Science, 1995, 268, 1860-1866.

44 M. M. Green, B. A. Garetz, B. Munoz, H. Chang, S. Hoke and R. G. Cooks, J. Am. Chem. Soc., 1995, 117, 4181-4182.

45 R. Fasel, M. Parschau and K.-H. Ernst, Nature, 2006, 439, 449452.

46 M. Parschau, S. Romer and K.-H. Ernst, J. Am. Chem. Soc., 2004, 126, 15398-15399.

47 D. C. Madden, M. L. Bentley, S. J. Jenkins and S. M. Driver, Surf. Sci., 2014, 629, 81-87.

48 F. Masini, N. Kalashnyk, M. M. Knudsen, J. R. Cramer, E. Lægsgaard, F. Besenbacher, K. V. Gothelf and T. R. Linderoth, J. Am. Chem. Soc., 2011, 133, 13910-13913.

49 A. Nuermaimaiti, C. Bombis, M. M. Knudsen, J. R. Cramer, E. Lægsgaard, F. Besenbacher, K. V. Gothelf and T. R. Linderoth, ACS Nano, 2014, 8, 8074-8081.

50 N. Katsonis, H. Xu, R. M. Haak, T. Kudernac, Z. Tomović, S. George, M. Van der Auweraer, A. P. H. J. Schenning, E. W. Meijer, B. L. Feringa and S. De Feyter, Angew. Chem. Int. Ed., 2008, 47, 4997-5001.

51 T. Chen, W.-H. Yang, D. Wang and L.-J. Wan, Nat. Commun., 2013, 4, 1389.

52 S.-Y. Li, T. Chen, J.-Y. Yue, L. Wang, H.-J. Yan, D. Wang and L.J. Wan, Chem. Commun., 2016, 52, 12088-12091.

53 I. De Cat, Z. Guo, S. J. George, E. W. Meijer, A. P. H. J. Schenning and S. De Feyter, J. Am. Chem. Soc., 2012, 134, 3171-3177.

54 Y. Fang, E. Ghijsens, O. Ivasenko, H. Cao, A. Noguchi, K. S. Mali, K. Tahara, Y. Tobe and S. De Feyter, Nat. Chem., 2016, 8, 711717.

55 A. Sanz-Matías, O. Ivasenko, Y. Fang, S. De Feyter, K. Tahara, Y. Tobe and J. N. Harvey, Nanoscale, 2018, 10, 1680-1694.

56 S. J. George, R. de Bruijn, Ž. Tomović, B. Van Averbeke, D. Beljonne, R. Lazzaroni, Albertus, P. H. J. Schenning and E. W. Meijer, J. Am. Chem. Soc., 2012, 134, 17789-17796.

57 I. Destoop, E. Ghijsens, K. Katayama, K. Tahara, K. S. Mali, Y. Tobe and S. De Feyter, J. Am. Chem. Soc., 2012, 134, 1955819571.

58 I. Destoop, A. Minoia, O. Ivasenko, A. Noguchi, K. Tahara, Y. Tobe, R. Lazzaroni and S. De Feyter, Faraday Discuss., 2017, 204, 215-231.

59 E. Ghijsens, H. Cao, A. Noguchi, O. Ivasenko, Y. Fang, K. Tahara, Y. Tobe and S. De Feyter, Chem. Commun., 2015, 51, 47664769.

60 Y. Fang, K. Tahara, O. Ivasenko, Y. Tobe and S. De Feyter, J. Phys. Chem. C, 2018, 122, 8228-8235.

61 E. Ghijsens, J. Adisoejoso, H. van Gorp, I. Destoop, A. Noguchi, O. Ivasenko, K. Tahara, M. Van der Auwaraer, Y. Tobe and S. De Feyter, J. Chem. Phys., 2015, 142, 101932.

62 J. Liu and C. Wöll, Chem. Soc. Rev., 2017, 46, 5730-5770.

63 M. Pivetta, M.-C. Blüm, F. Patthey and W.-D. Schneider, J. Phys. Chem. B, 2009, 113, 4578-4581.

64 A. Mairena, L. Zoppi, J. Seibel, A. F. Tröster, K. Grenader, M. Parschau, A. Terfort and K.-H. Ernst, ACS Nano, 2017, 11, 865871.

65 M. Parschau and K.-H. Ernst, Angew. Chem. Int. Ed., 2015, 54 , 14422-14426.

66 H. Cao and S. De Feyter, Nat. Commun., 2018, 9, 3416-3416.

67 P. Samori, N. Severin, C. D. Simpson, K. Müllen and J. P. Rabe, J. Am. Chem. Soc., 2002, 124, 9454-9457.

68 L. Piot, C. Marie, X. Feng, K. Müllen and D. Fichou, Adv. Mater. 2008, 20, 3854-3858.

69 Y. L. Huang, W. Chen and A. T. Wee, J. Am. Chem. Soc., 2011 $133,820-825$

70 D. Skomski, J. Jo, C. D. Tempas, S. Kim, D. Lee and S. L. Tait, Langmuir, 2014, 30, 10050-10056. 
71 J. Niederhausen, H. R. Kersell, C. Christodoulou, G. Heimel, H. Wonneberger, K. Müllen, J. P. Rabe, S. W. Hla and N. Koch, Langmuir, 2016, 32, 3587-3600.

72 A. Ciesielski, A. Cadeddu, C.-A. Palma, A. Gorczynski, V. Patroniak, M. Cecchini and P. Samori, Nanoscale, 2011, 3, 4125-4129.

73 M. O. Blunt, J. C. Russell, M. d. C. Gimenez-Lopez, N. Taleb, X. Lin, M. Schroder, N. R. Champness and P. H. Beton, Nat. Chem., 2011, 3, 74-78.

74 Y. Fang, B. Lindner, I. Destoop, T. Tsuji, Z. Zhang, R. Khaliullin, D. Perepichka, K. Tahara, S. De Feyter and Y. Tobe, J. Am. Chem. Soc., 2020, 142, 8662-8671.

75 T. Balandina, K. Tahara, N. Sandig, M. O. Blunt, J. Adisoejoso, S. B. Lei, F. Zerbetto, Y. Tobe and S. De Feyter, ACS Nano, 2012, 6, 8381-8389.

76 K. E. Plass, A. L. Grzesiak and A. J. Matzger, Acc. Chem. Soc., 2007, 40, 287-293.

77 K. Tahara, Y. Kubo, T. Ishikawa, S. Hashimoto, H. Kaneko, A. Brown, B. Hirsch, S. De Feyter and Y. Tobe, J. Am. Chem. Soc., 2020, 142, 7699-7708.

78 M. S. Dresselhaus, G. Dresselhaus and P. C. Eklund, Science of Fullerenes and Carbon Nanotubes: Their Properties and Applications, Academic Press, 1996.

79 R. Balog, B. Jørgensen, L. Nilsson, M. Andersen, E. Rienks, M. Bianchi, F. Fanetti, E. Lægsgaard, A. Baraldi, S. Lizzit, Z. Sljivancanin, F. Besenbacher, B. Hammer, T. G. Pedersen, P. Hofmann and L. Hornekær, Nat. Mater., 2010, 9, 315-319.

80 J. H. Jørgensen, A. C. Čabo, R. Balog, L. Kyhl, M. M. Groves, A. M. Cassidy, A. Bruix, M. Bianchi, M. Dendzik, M. A. Arman, L. Lammich, J. I. Pascual, J. Knudsen, B. Hammer, P. Hofmann and L. Hornekaer, ACS Nano, 2016, 10, 10798-10807.

81 J. J. Navarro, S. Leret, F. Calleja, D. Stradi, A. Black, R. Bernardo-Gavito, M. Garnica, D. Granados, A. L. Vázquez de Parga, E. M. Pérez and R. Miranda, Nano Lett., 2016, 16, 355361.

82 C. Galli, Chem. Rev., 1988, 88, 765-792.

83 M. Delamar, R. Hitmi, J. Pinson and J. M. Saveant, J. Am. Chem. Soc., 1992, 114, 5883-5884.

84 P. Allongue, M. Delamar, B. Desbat, O. Fagebaume, R. Hitmi, J. Pinson and J. M. Savéant, J. Am. Chem. Soc., 1997, 119, 201 207.

85 J. Greenwood, T. H. Phan, Y. Fujita, Z. Li, O. Ivasenko, W. Vanderlinden, H. van Gorp, W. Frederickx, G. Lu, K. Tahara, Y. Tobe, H. Uji-i, S. Mertens and S. De Feyter, ACS Nano, 2015, 9, 5520-5535.

86 K. Tahara, Y. Kubo, B. Lindner, S. Hashimoto, S. Hirose, A. Brown, B. E. Hirsch, L. Daukiya, S. De Feyter and Y Tobe, Langmuir, 2019, 35, 2089-2098.

87 Z. Xia, F. Leonardi, M. Gobbi, Y. Liu, V. Bellani, A. Liscio, A. Kovtun, R. Li, X. Feng, E. Orgiu, P. Samorì, E. Treossi and V. Palermo, ACS Nano, 2016, 10, 7125-7134.

88 V. Q. Nguyen, X. Sun, F. Lafolet, J.-F. Audibert, F. Miomandre, G. Lemercier, F. Loiseau and J.-C. Lacroix, J. Am. Chem. Soc. 2016, 138, 9381-9384.

89 A. Mattiuzzi, I. Jabin, C. Mangeney, C. Roux, O, Reinaud, L. Santos, F. Bergamini, P. Hapiot and C. Lagrost, Nat. Commun. 2012, 3, 1130.

90 K. Tahara, T. Ishikawa, B. E. Hirsch, Y. Kubo, A. Brown, S. Eyley, L. Daukiya, W. Thielemans, Z. Li, P. Walke, S. Hirose, S Hashimoto, S. De Feyter and Y. Tobe, ACS Nano, 2018, 12, 11520-11528.

91 Z. Li, H. Van Gorp, P. Walke, T. H. Phan, Y. Fujita, J. Greenwood, O. Ivasenko, K. Tahara, Y. Tobe, H. Uji-i, S. F. L. Mertens and S. De Feyter, Nanoscale, 2017, 9, 5188-5193.

92 M. M. Safont-Sempere, G. Fernández and F. Würthner, Chem. Rev., 2011, 111, 5784-5814.

93 M. L. Saha and M. Schmittel, Org. Biomol. Chem., 2012, 10, 4651-4684.
94 Z. He, W. Jiang and C. A. Schalley, Chem. Soc. Rev., 2015, 44, 779-789.

95 W. M. Bloch and G. H. Clever, Chem. Commun., 2017, 53 , 8506-8516.

96 M. Maeda, R. Nakayama, S. De Feyter, Y. Tobe and K. Tahara, Chem. Sci., 2020, 11, 9254-9261.

97 For a recent progress see: M. Yu, C. Chen, Q. Liu, C. Mattioli, H. Sang, G. Shi, W. Huang, K. Shen, Z. Li, P. Ding, P. Guan, S. Wang, Y. Sun, J. Hu, A. Gourdon, L. Kantorovich, F. Besenbacher, M. Chen, F. Song and F. Rosei, Nat. Chem., 2020, 12, 1035-1041.

98 F. Purcell-Milton, R. McKenna, L. J. Brennan, C. P. Cullen, L. Guillemeney, N. V. Tepliakov, A. S. Baimuratov, I. D. Rukhlenko, T. S. Perova, G. S. Duesberg, A. V. Baranov, A. V. Fedorov and Y. K. Gun'ko, ACS Nano, 2018, 12, 954-964. 